\title{
N-Terminal finger stabilizes the reversible feline drug GC376 in SARS-CoV-2 M$^{\text {pro }}$
}

Elena Arutyunova ${ }^{1,4}$, Muhammad Bashir Khan ${ }^{1}$, Conrad Fischer ${ }^{2 \dagger}$, Jimmy Lu ${ }^{1,4}$, Tess Lamer ${ }^{2}$, Wayne Vuong ${ }^{2}$, Marco J. van Belkum ${ }^{2}$, Ryan T. McKay ${ }^{2}$, D. Lorne Tyrrell ${ }^{3,4}$, John C. Vederas ${ }^{2}$, Howard S. Young ${ }^{1 *}$, M. Joanne Lemieux ${ }^{1,4 *}$

${ }^{1}$ Department of Biochemistry, Faculty of Medicine and Dentistry, Membrane Protein Disease Research Group, University of Alberta, Edmonton T6G 2R3, Alberta, Canada

${ }^{2}$ Department of Chemistry, Faculty of Science, University of Alberta, Edmonton T6G 2G2, Alberta, Canada

${ }^{3}$ Department of Medical Microbiology and Immunology, Faculty of Medicine and Dentistry, University of Alberta, Edmonton T6G 2E1, Alberta, Canada

${ }^{4} \mathrm{Li}$ Ka Shing Institute of Virology, Faculty of Medicine and Dentistry, University of Alberta, Edmonton T6G 2E1, Alberta, Canada

$\dagger$ Present address: Department of Physical Sciences, Barry University, 11300 NE 2nd Ave., Miami Shores FL 33161, USA

*Corresponding authors:

Howard S. Young: hyoung@ualberta.ca

M. Joanne Lemieux: mlemieux@ualberta.ca 
The main protease ( $\mathrm{M}^{\text {pro }}$, also known as 3CL protease) of SARS-CoV-2 is a high priority drug antiviral drug, GC376, has been shown to be effective in inhibiting the SARS-CoV-2 main

52 protease and live virus growth. As this drug moves into clinical trials, further characterization of

53 GC376 with the main protease of coronaviruses is required to gain insight into the drug's

54 properties, such as reversibility and broad specificity. Reversibility is an important factor for

55 therapeutic proteolytic inhibitors to prevent toxicity due to off-target effects. Here we demonstrate

56 that GC376 has nanomolar $\mathrm{K}_{\mathrm{i}}$ values with the $\mathrm{M}^{\text {pro }}$ from both SARS-CoV-2 and SARS-CoV

57 strains. Restoring enzymatic activity after inhibition by GC376 demonstrates reversible binding

58 with both proteases. In addition, the stability and thermodynamic parameters of both proteases

59 were studied to shed light on physical chemical properties of these viral enzymes, revealing higher

60 stability for SARS-CoV-2 $\mathrm{M}^{\text {pro }}$. The comparison of a new X-ray crystal structure of $\mathrm{M}^{\text {pro }}$ from

61 SARS-CoV complexed with GC376 reveals similar molecular mechanism of inhibition compared

62 to SARS-CoV-2 Mpro, and gives insight into the broad specificity properties of this drug. In both

63 structures, we observe domain swapping of the N-termini in the dimer of the $\mathrm{M}^{\text {pro }}$, which facilitates

64 coordination of the drug's P1 position. These results validate that GC376 is a drug with an off-rate

65 suitable for clinical trials.

67 Keywords: 3CL protease, coronavirus, proteolytic inhibitor, COVID-19, antivirals, enzyme mechanism, SARS, GC373

69 


\section{Introduction}

In late 2019, a respiratory infection initially detected in China, was sparking fear of a viral

73 outbreak [1]. This respiratory infection attributed to severe acute respiratory syndrome coronavirus

742 (SARS-CoV-2), led to an ongoing coronavirus disease 2019 (COVID-19) pandemic with

75 millions infected worldwide (https://coronavirus.jhu.edu/map.html). This respiratory illness was

76 similar to a previous infection by SARS-CoV that led to a SARS outbreak in 2002/3 as well as the

77 Middle East respiratory infection (MERS) outbreak in 2012 [2,3]. All of these outbreaks stem from

78 related betacoronavirus infections, suggesting these strains will likely lead to future viral

79 outbreaks. Vaccines have been developed and will be important for prevention of new infections

80 in the future. However, even with a $95 \%$ immunity rate, there will be a significant proportion of

81 people worldwide who will require therapeutic treatment. Antiviral development remains a priority

82 because of importance of immediate mitigation of acute infections, vaccine hesitancy, and the

83 inability to vaccinate some individuals. The outbreak of SARS in 2003 and MERS in 2012 along

84 with the current pandemic reminds us that pan-inhibitors may provide a means for initial control

85 of outbreaks, thereby preventing or quickly controlling pandemics in the future [4].

SARS-CoV-2 is a 30-kb positive-sense single-stranded RNA virus that is translated by the

87 host's cellular machinery to generate two alternatively spliced long polypeptides, PP1a and PP1ab.

88 These long polypeptides release non-structural proteins (nsps), including the RNA-dependent

89 RNA polymerase, that are essential for viral replication after proteolytic cleavage by proteases

90 from domain nsp3 and nsp5, respectively, a papain-like (PL $\left.{ }^{\text {pro }}\right)$ protease and a chymotrypsin-like

91 main protease $\left(\mathrm{M}^{\text {pro }}\right.$ or $\left.3 \mathrm{CL}^{\mathrm{pro}}\right)[5]$. Similar to SARS-CoV, the SARS-CoV-2 M ${ }^{\text {pro }}$ enzyme

92 recognises the sequence of Leu-Gln $\downarrow$ Ser-Ala-Gly, where $\downarrow$ marks the cleavage site and this

93 sequence is widely employed for generation of substrates for kinetic analysis and for development 
94 of peptidomimetic specific probes and inhibitors [6] [7,8]. The essential role of the $\mathrm{M}^{\text {pro }}$ in viral

95 replication has resulted in a great deal of crystallographic and in silico studies working towards

96 the development of antiviral therapies to treat COVID-19 [9-13].

97 Proteolytic inhibitors have been used successfully as antiviral therapeutics [14]; for

98 example peptidomimetic inhibitors for the human immunodeficiency virus (HIV) protease and

99 small molecule inhibitors for hepatitis C virus (HCV) protease. The HIV protease inhibitors, along

100 with other drugs, are used in a combination therapy and play a big role in the treatment of

101 symptoms and the subsequent reduction in spread of infection.

102 It has been recently shown by our group, as well as by other teams, that M ${ }^{\text {pro }}$ of SARS-

$103 \mathrm{CoV}-2$ is a promising drug target for the development of SARS-CoV-2 antivirals $[10,11,13,15]$.

104 We demonstrated that the proteolytic inhibitor GC376 (a bisulphite prodrug) used to treat feline

105 coronavirus infection and its related aldehyde inhibitor, GC373, are effective at decreasing viral

106 load of SARS-CoV-2 in cell culture [13]. These drugs have previously been shown to be effective

107 inhibiting the $\mathrm{M}^{\text {pro }}$ of picornavirus, norovirus and coronavirus, and furthermore have been

108 validated in animal models for both SARS and MERS [16-18]. Even though we have a

109 considerable understanding of the efficacy of GC376 and GC373 with both SARS-CoV and

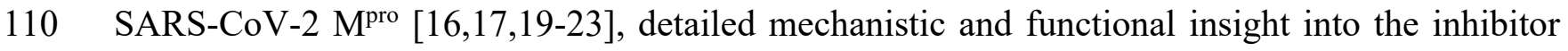

111 binding process is still essential for directing broad-spectrum inhibitors in clinical trials. For

112 example, one of desirable features for peptidomimetic proteolytic inhibitors is the reversible nature

113 of binding since it reduces the risk of strong off-target effects and potential toxicity [24,25]. In

114 addition, in light of the new variants, we need a clear understanding of the efficacy of GC373 and

115 GC376 with other coronavirus $\mathrm{M}^{\text {pro }}$, and importantly a crystal structure of these inhibitors with the

116 SARS-CoV M $\mathrm{M}^{\text {pro }}$ has not been determined. 
118 GC376 using kinetic and structural approaches. We determine $\mathrm{K}_{\mathrm{i}}$ values are in the low nanomolar

119 range for both SARS-CoV and SARS-CoV-2 M ${ }^{\text {pro }}$. After inhibition with GC376, NMR and activity

120 assays demonstrate the reversible nature of inhibition for both proteases. In addition, the

121 restoration of activity of $\mathrm{M}^{\text {pro }}$ after inhibition reveal a high kinetic and thermodynamic stability for

122 these viral proteases. We determine the crystal structures of SARS-CoV M ${ }^{\text {pro }}$ inhibited with the

123 dipeptidyl inhibitor, GC376, and aldehyde form, GC373, both of which reveal a covalent mode of

124 inhibition similar to SARS-CoV-2 $\mathrm{M}^{\text {pro }}$. We highlight in both structures the role of the N-terminus

125 in stabilizing the S1 subsite from domain swapping, and how this facilitates drug binding. This

126 comparative analysis of $\mathrm{M}^{\text {pro }}$ from SARS-CoV and SARS-CoV-2 provides additional insight into

127 the mechanism of inhibition by this anti-coronaviral drug.

129 Results

$130 \mathbf{K}_{\mathbf{i}}$ values of GC376 inhibition of $M^{\text {pro }}$ from both SARS-CoV and SARS-CoV-2 are in

131 nanomolar range

132 Determining $\mathrm{K}_{\mathrm{i}}$ values that are reflective of drug binding affinity is a prerequisite for the

133 prediction and evaluation of drug interactions. In our previous report, we determined the half-

134 maximal inhibitor concentrations $\left(\mathrm{IC}_{50}\right)$, values, which describe the functional strength of the

135 inhibitor, the feline drug GC376 with both $\mathrm{M}^{\text {pro }}$ of SARS-CoV and SARS-CoV-2 [13]. Here we

136 determine $\mathrm{K}_{\mathrm{i}}$ values for the prodrug GC376 with both $\mathrm{M}^{\text {pro }}$ of SARS-CoV and SARS-CoV-2. For

$137 \mathrm{~K}_{\mathrm{i}}$ determination, the inhibitory effects of increasing concentrations of GC376 on $\mathrm{M}^{\text {pro }}$ from both

138 SARS-CoV and SARS-CoV-2 were tested using the synthetic peptide FRET-substrate Abz-

139 SVTLQSG-Y $\left(\mathrm{NO}_{2}\right)-\mathrm{R}$ followed by Michaelis-Menten kinetics. Data was plotted as reaction rate 
140 versus substrate concentration (primary Lineweaver-Burk plot) and the slopes (Km/Vmax) were

141 determined by linear regression analysis. The slopes were plotted versus the concentration of

$142 \mathrm{GC} 376$ to determine the inhibitory constant ( $\mathrm{K}_{\mathrm{i}}$ as $\mathrm{y}$-intercept). The $\mathrm{K}_{\mathrm{i}}$ for GC376 was $0.02 \mu \mathrm{M}$

143 for SARS-CoV M $\mathrm{M}^{\text {pro }}$ and $0.04 \mu \mathrm{M}$ for SARS-CoV-2 $\mathrm{M}^{\text {pro }}$, (Fig 1 and Table 1).

\section{GC376 is a reversible inhibitor with $M^{\text {pro }}$ from both SARS-CoV and SARS-CoV-2}

An important factor to consider when developing a therapeutic protease inhibitor is the

147 reversibility of compound binding [24]. Irreversible protease drugs can yield long-lasting effects

148 by permanently blocking proteases in cells that are not the intended target and thus causing

149 detrimental consequences resulting in side effects and antigenicity of covalently modified proteins

150 [26]. We previously demonstrated that the bisulfite prodrug GC376 converts to the peptide

151 aldehyde GC373 which interacts covalently with the catalytic cysteine of SARS-CoV-2 M ${ }^{\text {pro }}[13]$,

152 but did not assess experimentally whether the inhibition was reversible.

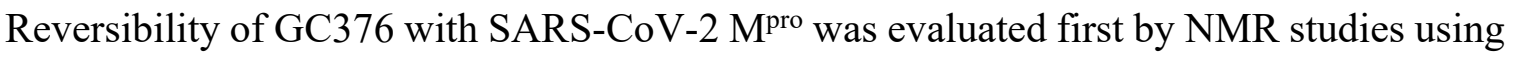

$154{ }^{13}$ C-labelled GC373 (Fig 2A). HSQC experiments of samples containing only SARS-CoV-2 Mpro

155 (Fig 2B), inhibitor (Fig 2C), or both co-incubated (Fig 2D) provided spectra to which the

156 reversibility experiment could be compared. Evidence of binding reversibility was acquired by

157 HSQC experiments conducted on a co-incubated sample containing both enzyme and inhibitor that

158 was subsequently washed with buffer. The subsequent HSQC experiment using this sample

159 showed a disappearance of the NMR signal corresponding to the bound inhibitor (Fig $\mathbf{2 E}$ ). The

160 disappearance of this signal would only be observed in the case of a binding reversion event.

We then conducted a detailed study to provide the rate and percentage of reversibility, as

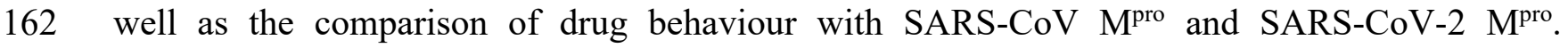


163 Reversibility was tested by measuring catalytic activity post dialysis. Incubation of SARS-CoV

$164 \mathrm{M}^{\text {pro }}$ and SARS-CoV-2 $\mathrm{M}^{\text {pro }}$ with the GC376 followed by dialysis resulted in increase in enzymatic

165 activity over time, indicative of a reversible dissociation of inhibitor (Fig. 3). We observed a

166 recovery of $10 \%$ of activity after 22 hours of dialysis, which reached $30-40 \%$ of initial activity

167 for SARS-CoV and 40-60\% for SARS-CoV-2 after 4 days of dialysis, suggesting over time the

168 substrate competed for the enzyme binding site. To ensure the proteins remained stable over this

169 time period, we also monitored the stability of uninhibited enzymes, which was compared with the

170 activity of recovered enzymes. After 4 days the residual protease activity for the uninhibited $\mathrm{M}^{\text {pro }}$

171 of SARS-CoV and SARS-CoV-2 was 30-40\%, which allowed us to conclude that the drug was

172 fully reversible.

173

174 SARS-CoV-2 Mpro has enhanced stability compared to SARS-CoV Mpro

175 After observing the high kinetic stability of both viral proteases at room temperature, we

176 characterized their thermal stability and assessed their thermodynamic parameters including

177 activation energies of inactivation. Thermal stability is a characteristic used to describe the kinetic

178 stability of enzymes, and many individual proteins or protein complexes are known to have high

179 kinetic stability [27-31]. For viral proteins, particularly the structural ones, this feature is crucial

180 because virus particles must be able to resist harsh environmental conditions until they find a new

181 host to infect and also remain stable during infection [10,13,32]. For example, determination of

182 thermodynamic parameters of the HIV protease in the presence of various inhibitors was used to

183 reveal the differences in protein stability upon forming inhibitor-protein complexes, which

184 informed on inhibitor design [33]. 

and 4F) was studied at the temperature range of $24-70{ }^{\circ} \mathrm{C}$ in a time-dependant manner. The

187 semilogarithmic plots of residual activity versus incubation time were linear at all temperatures

188 for both proteins, which was indicative of a simple first-order monophasic kinetic process. From

189 the slopes of semilogarithmic plots inactivation rate constants were calculated and are given in

190 Table 2. For both proteases, the rate constant progressively increased with increasing temperatures

191 whereas half-life $\left(t_{1 / 2}\right)$ and the decimal reduction time $(\mathrm{Dt})$, two important parameters used in

192 characterization of enzyme stability, decreased.

The dependence of inactivation rate constants on temperature was plotted using the

194 Arrhenius equation (Fig 4C and 4F), from which apparent activation energies of inactivation (Ea)

195 were calculated. Interestingly, Arrhenius plots for both proteases were not linear and showed 196 upward curvature suggesting two denaturation processes each with its own temperature

197 dependence and activation energy. At temperatures above $37^{\circ} \mathrm{C}$ inactivation is a result of protein 198 unfolding with high activation energy, with the rate of this process strongly dependant on 199 temperature. At temperatures of $37{ }^{\circ} \mathrm{C}$ and below this rate becomes insignificant and other 200 processes with low activation energy prevail. The activation energies for the high temperature 201 range were found to be high and similar for SARS-CoV $\mathrm{M}^{\text {pro }}(\mathrm{Ea}=243.6 \mathrm{~kJ} / \mathrm{mol})$ and SARS-CoV$2022 \mathrm{M}^{\text {pro }}(\mathrm{Ea}=234.2 \mathrm{~kJ} / \mathrm{mol})$. However, for the low temperature range the activation energies were $203 \quad 10-20 \%$ of those determined at high temperature, confirming that $\mathrm{M}^{\text {pro }}$ inactivation involves both 204 high- and low-activation energy processes. Interestingly, the parameters of the inactivation process 205 at low temperature range $\left(24-37^{\circ} \mathrm{C}\right)$ are different for $\mathrm{M}^{\text {pro }}$ from SARS-CoV and SARS-CoV-2, 206 showing Ea of $16.4 \mathrm{~kJ} / \mathrm{mol}$ and $41.4 \mathrm{~kJ} / \mathrm{mol}$ and $t_{1 / 2}\left(\right.$ at $24{ }^{\circ} \mathrm{C}$ ) of $38.5 \mathrm{~h}$ and $57.7 \mathrm{~h}$ respectively, 207 suggesting higher stability for SARS-CoV M pro. $^{\text {pro }}$ 
Determination of all thermodynamic parameters of inactivation can provide further

209 information on enzyme stability. $\Delta \mathrm{G}$ value, the Gibbs free energy, which is the energy barrier for

210 enzyme inactivation, is directly related to protein stability. We see a significant decrease in $\Delta \mathrm{G}$ for

211 the temperatures above $55{ }^{\circ} \mathrm{C}$ indicating that the destabilization process occurs rapidly in this

212 temperature range (Table 2).

213 To gain a deeper insight into the driving forces of SARS-CoV M ${ }^{\text {pro }}$ and SARS-CoV-2 M pro $^{\text {pro }}$

214 stability, the Gibbs free energy was decomposed into its enthalpic and entropic contributions.

215 Enthalpy, $\Delta \mathrm{H}$, measures the number of non-covalent bonds broken during transition state

216 formation for enzyme inactivation, allowing us to compare the energy landscapes of both SARS-

$217 \mathrm{CoV} \mathrm{M}^{\text {pro }}$ and SARS-CoV-2 $\mathrm{M}^{\text {pro }}$. For temperature ranging from $37{ }^{\circ} \mathrm{C}$ to $70{ }^{\circ} \mathrm{C}$ we observed

218 consistent high $\Delta \mathrm{H}$ values, which is in agreement with a temperature-dependent inactivation

219 process. Interestingly, at the $24{ }^{\circ} \mathrm{C}$ and $37^{\circ} \mathrm{C}$ temperature interval a significant jump in $\Delta \mathrm{H}$ occured

220 for both proteases, however, with different initial enthalpy values for SARS-CoV M ${ }^{\text {pro }}$ and SARS-

$221 \mathrm{CoV}-2 \mathrm{M}^{\text {pro }}$ at $24{ }^{\circ} \mathrm{C}(13.9$ and $38.9 \mathrm{~kJ} / \mathrm{mol}$ respectively), again highlighting higher stability of

222 latter at physiological temperatures (Table 2). The compactness in the protein molecular structure

223 as well as enzyme and solvent disorder can be inferred through the quantitative analysis of entropy

$224 \Delta \mathrm{S}$ values $[34,35]$. Small negative entropy values at $24{ }^{\circ} \mathrm{C}$ for both SARS-CoV $\mathrm{M}^{\text {pro }}$ and SARS-

$225 \mathrm{CoV}-2 \mathrm{M}^{\text {pro }}$ confirmed no disorder in protein structure upon inactivation; however, at higher

226 temperatures all values of $\Delta \mathrm{S}$ were positive and similar, suggesting that unfolding is a rate-limiting

227 step at this range (Table 2). 
We previously reported increased catalytic activity of SARS-CoV-2 $\mathrm{M}^{\text {pro }}$ in comparison to

231 SARS-CoV $\mathrm{M}^{\text {pro }}$ with the catalytic turnover rate being almost 5 times higher for the former using

232 a FRET-peptide as substrate [13]. We were interested in structural comparison of the $\mathrm{M}^{\text {pro }}$ from

233 SARS-CoV and SARS-CoV-2, for both apo and drug-bound forms to reveal differences that

234 account for the enhancement in activity. Crystal structures of apo-M ${ }^{\text {pro }}$ from SARS-CoV and

235 SARS-CoV-2, and bisulphite prodrug (GC376) and the aldehyde drug (GC373) bound forms were

236 determined. The two proteins share $96 \%$ sequence identity with only 12 out of 306 residues being

237 different (S1 Fig). Therefore, as expected, there is little change in the overall structures of apo-

238 SARS-CoV and SARS-CoV-2 Mpro (Fig 5), with an RMSD of $0.6 \AA$. We observed a new helical

239 feature at $\eta 2$ (residues 47-50) in SARS-CoV-2, which is unfolded in SARS-CoV, (S1 and S2 Fig).

240 It is located at the entrance to the active site, near a non-conserved residue between SARS-CoV,

241 and SARS-CoV-2 (S2 Fig). In the GC373-bound form of proteins, however we observed the

242 opposite; this helix is found in the $\mathrm{M}^{\text {pro }}$ of SARS-CoV but not in SARS-CoV-2 (S3 Fig), suggesting

243 a dynamic nature of this structural element.

244 Both SARS-CoV and SARS-CoV-2 $\mathrm{M}^{\text {pro }}$ form dimers, and while monomers have very low

245 activity dimerization is necessary for full enzymatic activity and virulence [36,37]. Comparative

246 analysis of the biological dimer of the two proteases revealed that the main differences are located

247 at the dimer interface. In the $\mathrm{M}^{\text {pro }}$ of SARS-CoV-2, we observed a slight shift of the chymotrypsin-

248 like domains away from each other, compared to the $\mathrm{M}^{\text {pro }}$ of SARS-CoV (Fig 5B). However, the

249 biggest change is the difference in association between the dimerization domains (Fig 5C and

250 5D). The dimer interface of SARS-CoV and SARS-CoV-2 $M^{\text {pro }}$ is facilitated by several

251 interactions between the two protomers, one of which is between the helical domain III of each

252 protomer comprising of residues 284-286, specifically Ser-Thr-Ile (STI) in SARS-CoV M ${ }^{\text {pro }}$ and 
253 Ser-Ala-Leu (SAL) in SARS-CoV-2 $\mathrm{M}^{\text {pro. }}$. This unstructured loop self-associates between

254 protomers in the dimer. Importantly, this region harbors a non-conservative residue in sequence at

255 the dimer interface, where the Thr285 in SARS-CoV M ${ }^{\text {pro }}$ is altered to Ala285 in SARS-CoV-2

$256 \mathrm{M}^{\text {pro }}$ (Fig 5E and 5F). The SAL-motif forms a tight van der Waals interaction and the residues

257 from each protomer interdigitate to form a complementary interface that readily explains the

258 observed enhanced stability.

GC376 inhibited forms of SARS-CoV-2 and SARS-CoV Mpro reveal a common mechanism

of inhibition

We recently presented the structure of GC373 with the SARS-CoV-2 Mro [13]. The

263 structure of SARS-CoV-2 $\mathrm{M}^{\text {pro }}$ with drug GC373, as well as prodrug GC376 that converts to

264 GC373, reflects the specificity of the enzyme for a glutamine surrogate in the P1 position and a

265 leucine, which is preferred in the P2 position. A benzyl group is in the P3 position. Here we

266 determined the crystal structure of the SARS-CoV M ${ }^{\text {pro }}$ with the prodrug GC376 and drug GC373

267 to examine features that determine its efficacy and compare this with the previously determined

268 SARS-CoV-2 structure (Fig 6).

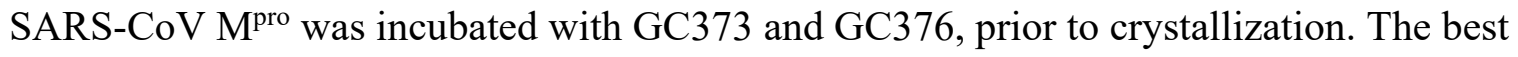

270 crystals diffracted to $2.0 \AA$, and the data was refined with good statistics (Table 3). Overall

271 comparison of SARS-CoV M $M^{\text {pro }}$ and SARS-CoV-2 M ${ }^{\text {pro }}$ structures with GC373 showed similar

272 agreements with the apo-M $\mathrm{M}^{\text {pro }}$ structures, with an RMSD of $0.6 \AA$ (Fig 6). The drug binding is

273 supported by H-bonding with the main chain of oxyanion hole residues Asn142, Gly143 and

274 Ser144, which are identical for both proteases (Fig 6B, S4 Fig and S5 Fig). A good fit was

275 observed for both the P1 and P2 positions, supported structurally by hydrogen bonding and van 
276 der Waals interactions respectively with $\mathrm{H}$-bonds for the $\mathrm{P} 1$ position being identical for $\mathrm{M}^{\text {pro }}$ from

277 SARS-CoV and SARS-CoV-2 (Fig 6C, S4 Fig and S5 Fig).

278

279 The N-terminal finger of the $\mathbf{M}^{\text {pro }}$ stabilizes dimer formation and coordination of the drug

$280 \quad$ GC373

A distinctive feature of $\mathrm{M}^{\text {pro }}$ dimer is the interaction of $\mathrm{N}$-terminal residues ("N-finger") of

282 protomer A with residues of domain II of protomer B. In the dimer for both protomers of SARS-

283 CoV-2 $\mathrm{M}^{\text {pro }}$ and SARS-CoV $\mathrm{M}^{\text {pro }}$, we observe the N-termini interact with residues near S1

284 substrate-binding subsite in a hairpin adjacent to the oxyanion hole of the active site (Fig 7). The

285 NH-group of Ser1 from protomer A forms strong H-bonds with the carboxylate group of Glu166

286 (3.1 $\AA$ ) and the carbonyl of Phe140 (3.3 $\AA)$ of protomer B and vice versa. This interaction stabilizes

287 the enzyme, assists in the correct orientation of the oxyanion loop and S1 pocket of the substrate

288 binding site, and thus results in enhanced catalytic efficiency, as observed in previous studies

289 demonstrating the native N-terminal serine provides the most efficient enzyme with SARS-CoV

$290 \mathrm{M}^{\text {pro }}$ [38]. Interestingly, the H-bond distance between the Ser1 (protomer A) and Phe140 (protomer

291 B) is closer in SARS-CoV-2 Mpro (3.3 $\AA)$ compared to SARS-CoV Mpro (5.5 A) (Fig. 8), likely

292 adding to its increased catalytic activity. The proper conformation of S1 pocket is also important

293 for the drug binding and importantly, P1 position of GC373 is also stabilized by hydrogen bonding

294 between the side chain of Glu166 (3.3 $\AA)$ and backbone carbonyl of Phe140 (3.3 $\AA)$ residues (Fig

295 8). Thus, a hydrogen bond network between the dimer in $\mathrm{M}^{\text {pro }}$ stabilizes the $\mathrm{S} 1$ substrate for

296 substrate binding and hence inhibitor binding.

297 Residues adjacent to the N-terminus also play a key role in dimerization, specifically Pro9

298 and Phe305 from protomer A, which interact with residues Pro122 and Ser123 in a strand on 
protomer B. We also observe these interactions in all of our SARS-CoV M ${ }^{\text {pro }}$ and SARS-CoV-2 $\mathrm{M}^{\text {pro }}$ structures bound to the inhibitor (S7 Fig). Mutation of Pro9 to Thr results in a monomeric species of SARS-CoV-2 Mro [39]. Together this data suggests a strong role for the N-terminus of the protease not only in function and stability, but also with inhibitor coordination.

\section{Discussion}

Here we show that the feline antiviral prodrug GC376 is reversible and inhibits $\mathrm{M}^{\text {pro }}$ of both SARS-CoV and SARS-CoV-2 with low nanomolar $\mathrm{K}_{\mathrm{i}}$ values. While $\mathrm{IC}_{50}$ values, the concentration of inhibitor at half-maximal inhibition, are very useful during drug development [40], $\mathrm{K}_{\mathrm{i}}$ values describe precise binding affinity between the inhibitor and enzyme, independent of experimental conditions, and allow for comparisons during structure-activity relationship (SAR) studies. Here we show $\mathrm{K}_{\mathrm{i}}$ values for GC376 with the SARS-CoV and SARS-CoV-2 M $\mathrm{M}^{\text {pro }}$ to be 20

$311 \mathrm{nM}$ and $40 \mathrm{nM}$, respectively. These are lower, as expected, when compared to the $\mathrm{IC}_{50}$ values of 312 prodrug GC376 (190 nM) and drug GC373 (400 nM) with SARS-CoV-2 M Mro $^{\text {[13]. The high }}$ 313 degree of sequence identity between the SARS-CoV and SARS-CoV-2 M $^{\text {pro }}$ suggests strong 314 conservation in proteolytic inhibition supported by $\mathrm{K}_{\mathrm{i}}$ values. $\mathrm{K}_{\mathrm{i}}$ values for GC376 are in line with $\mathrm{K}_{\mathrm{i}}$ values of reported proteolytic inhibitors targeting

316 the HCV serine protease and currently being used to treat hepatitis $\mathrm{C}$ such as first-generation $\mathrm{HCV}$

317 ns3/4A inhibitors Boceprevir with low $\mathrm{nM} \mathrm{K} \mathrm{K}_{\mathrm{i}}$ values and second-generation inhibitors with 318 subnanomolar $\mathrm{K}_{\mathrm{i}}$ values [41]. These drugs are reversible serine protease inhibitors whose 319 development was facilitated by SAR studies [41,42]. Our $\mathrm{K}_{\mathrm{i}}$ data further supports GC376 being a 320 broad-spectrum inhibitor $[16,17,20,22]$, and demonstrates it is in the inhibitory range to be 321 considered as a viable antiviral for clinical trials. 

with the exception of Ala285 discussed above, are conservative (S1 Fig). Therefore, it was not

324 surprising that both proteins revealed similar physical chemical properties such as high thermal

325 stability at temperatures above $37^{\circ} \mathrm{C}$ with high activation energies and enthalpy independent of

326 temperature (Table 2). However, at physiological temperatures $\left(24-37{ }^{\circ} \mathrm{C}\right)$ we observed a 327 difference in stability between SARS-CoV $\mathrm{M}^{\text {pro }}$ and SARS-CoV-2 M $\mathrm{M}^{\text {pro }}$, with the latter being more 328 stable, exhibiting higher values of $\mathrm{t}_{1 / 2}\left(38.5 \mathrm{~h}\right.$ for SARS-CoV M $\mathrm{M}^{\text {pro }}$ versus $57.7 \mathrm{~h}$ for SARS-CoV$3292 \mathrm{M}^{\text {pro }}$ ) and enthalpy (13.9 kJ/mol for SARS-CoV M $\mathrm{M}^{\text {pro }}$ versus $38.9 \mathrm{~kJ} / \mathrm{mol}$ for SARS-CoV-2 $\mathrm{M}^{\text {pro }}$ ).

330 A high $\Delta \mathrm{H}$ value is usually indicative of a larger number of noncovalent intramolecular bonds, 331 which contribute to protein stability. Therefore, in order to understand what variant residues could 332 be responsible for enhanced stability of SARS-CoV-2 $\mathrm{M}^{\text {pro }}$ compared to SARS-CoV, we 333 examined the regions with amino acid substitutions more closely. of SARS-CoV M $\mathrm{M}^{\text {pro }}$ elucidated how the dimers assemble $[7,43]$ and mutagenesis has revealed that

336 residues at the dimer interface are important for both activity and stability [36,37,44]. From our 337 crystal structures we observe that overall the dimerization motifs of both SARS-CoV and SARS-

338 CoV-2 $\mathrm{M}^{\text {pro }}$ are very similar, however, one key change at the domain III interface, namely 339 Thr285Ala in SARS-CoV-2 Mro, results in a significant alteration in the distance between the

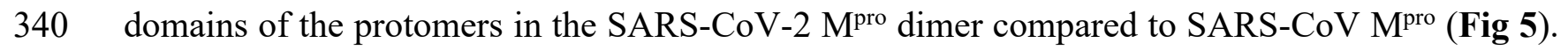

341 This mutation leads to residues in the domain III interface forming a hydrophobic zipper clearly 342 aligning the two domains, and thus likely enhancing the $t_{1 / 2}$ at low temperatures as we have 343 observed above. The high degree of stability of the enzymes for both SARS-CoV and SARS-CoV3442 is an interesting feature that likely contributes to viral potency. 
Another structural feature that might explain the increased activity and stability is a closer association between the N-finger Ser1 and Phe140 in the oxyanion loop in the $\mathrm{M}^{\text {pro }}$ of SARS-CoV-

3472 compared to SARS-CoV (Fig 8). This interaction plays a critical role for activity since it sustains

348 the correct conformation of the oxyanion loop, therefore precise coordination of the $\mathrm{N}$-finger in

349 both $\mathrm{M}^{\text {pro }}$ of SARS-CoV and SARS-CoV-2 is a prerequisite for function. Previous work

350 demonstrated that enzymatic activity of SARS-CoV $\mathrm{M}^{\text {pro }}$ was diminished with non-native affinity

351 tags proving the need for native $\mathrm{N}$ - and C-termini [6,38]. The effect was most pronounced with

352 additional residues at the N-terminus, with the activity of the wild-type being 20 -fold greater than

353 a variant with an additional glycine at the N-terminus [38].

354 While GC376 has been crystallized with the main protease of the similar betacoronavirus

355 MERS [18], as well as other viral proteases, including norovirus and porcine diarrhea virus

356 (PEDV) [45], no N-finger association was observed in those crystal structures. This structural

357 motif, however, was observed in a SARS-CoV $\mathrm{M}^{\text {pro }}$ crystal structure with a Michael acceptor

358 inhibitor, however the N-finger interaction was diminished with the addition of residues at the

359 native $\mathrm{N}$-terminus [38].

360 We demonstrated that the NH group of Ser 1 donates H-bonds to Phe140 and Glu166, the

361 residues that coordinate the $\mathrm{N}$-termini of each protomer in the dimer. Importantly, these residues

362 also interact with the P1 position of GC373 in both SARS-CoV and SARS-CoV-2, demonstrating

363 a strong hydrogen bond network near the active site, and stabilization of the S1 subsite pocket.

364 This likely contributes to the high $\mathrm{K}_{\mathrm{i}}$ values for these inhibitors. The precise structural and 365 mechanistic elucidation of the inhibitor-protease interaction and implications for $\mathrm{M}^{\text {pro }}$ dimerization

366 is paramount for the fine-tuned design of universally active inhibitor drugs. In this regard, the 
367 current study provides a rationale for the precise nature of a gamma-lactam group in the P1 position 368 of the GC373/GC376 inhibitor.

With coronavirus outbreaks occurring in 2002, 2015 and 2019, it is clear that broad-

370 spectrum antivirals will be needed for the current pandemic and in the future. The development of

371 antivirals to treat coronavirus infections remains a high priority. By comparing kinetic,

372 thermodynamic, and structural features of $\mathrm{M}^{\text {pro }}$ from SARS-CoV and SARS-CoV-2 and their

373 binding to GC373/GC376 we revealed distinct supramolecular differences in overall protease

374 properties, yet demonstrate comparable efficacies of GC376 with both proteases. Furthermore,

375 reversible inhibition with the drug further supports the clinical potential of the GC376 compound.

376 The results presented here support the use of GC376 as an antiviral with broad specificity against

377 coronaviruses.

379 Methods

381 Purifications of proteases were performed as described earlier [13]. Briefly, pET SUMO (small 382 ubiquitin-like modifier) expression vector (Invitrogen) bearing $\mathrm{M}^{\text {pro }}$ from SARS-CoV-2 gene with 383 N-terminal His-SUMO tag was transformed into E. coli BL21 (DE3), induced with $0.5 \mathrm{mM}$ 384 isopropyl $\beta$-d-1-thiogalactopyranoside and the protein was expressed for $4-5 \mathrm{~h}$ at $37^{\circ} \mathrm{C}$. After 385 harvesting by centrifugation $\left(4400 \times g\right.$ for $10 \mathrm{~min}$ at $\left.4^{\circ} \mathrm{C}\right)$ cells were suspended in lysis buffer 386 (20 mM Tris- $\mathrm{HCl}, \mathrm{pH} 7.8,150 \mathrm{mM} \mathrm{NaCl}, 5 \mathrm{mM}$ imidazole) and lysed by sonication. The lysate 387 was clarified by centrifugation at $17,000 \times g$ for $30 \mathrm{~min}$, and the supernatant was loaded onto Ni388 NTA resin column (Qiagen). The resin was washed with 10 column volumes of lysis buffer 389 containing $20 \mathrm{mM}$ imidazole and the fusion protein was eluted with $40-500 \mathrm{mM}$ imidazole in the 
same buffer. Eluted fractions containing the protein of interest were pooled together and dialyzed

391 against lysis buffer containing $1 \mathrm{mM} \mathrm{DTT}$ at $4{ }^{\circ} \mathrm{C}$. The fusion protein was subsequently digested

392 with His-tagged SUMO protease (McLab, South San Francisco, CA) at $4{ }^{\circ} \mathrm{C}$ for $1-2 \mathrm{~h}$ to remove

393 the SUMO tag and the resulting cleavage mixture was then passed through Ni-NTA resin column.

394 The flow through containing SARS-CoV-2 Mpro was collected and further purified using size

395 exclusion chromatography column (G-100, GE Healthcare,) equilibrated with $20 \mathrm{mM}$ Tris, $20 \mathrm{mM}$

$396 \mathrm{NaCl}, 1 \mathrm{mM}$ DTT, $\mathrm{pH}$ 7.8. Fractions containing the SARS-CoV-2 $\mathrm{M}^{\text {pro }}$ protein were pooled and

397 concentrated using Amicon Ultra-15 filter with a MWCO of $10 \mathrm{kDa}$. The plasmid encoding the

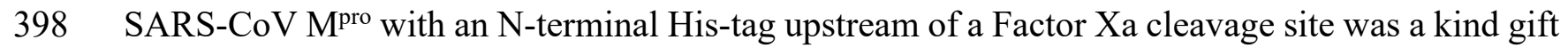

399 of Dr. Michael James. The protein was expressed and purified the same way as SARS-CoV Mpro_

4002 but Factor Xa protease (Sigma, Canada) was used $\left(4{ }^{\circ} \mathrm{C}\right.$, overnight) to remove the tag.

Inhibitor and FRET Substrate Synthesis

403 Inhibitors GC373 and GC376, and the FRET substrate Abz-SVTLQSG-Y(NO 2$)-\mathrm{R}$ were 404 synthesized according to methods previously described[13].

\section{Kinetic experiments}

407 The activity determination of both proteases was performed as previously described[13] using 408 FRET-based cleavage assay with a synthesized fluorescent substrate containing the cleavage site 409 (indicated by the arrow, $\downarrow$ ) of SARS-CoV-2 M pro (Abz-SVTLQ $\downarrow$ SG-Tyr(NO 2 -R) in 20 mM Bis-

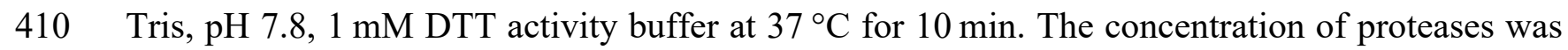

411 fixed at $80 \mathrm{nM}$ and the range of $0.1-500 \mu \mathrm{M}$ was used for the substrate. Reactions were started 412 with the enzyme and the fluorescence signal of the Abz-SVTLQ peptide cleavage product was 
413 monitored at an emission wavelength of $420 \mathrm{~nm}$ with excitation at $320 \mathrm{~nm}$, using an Flx 800

414 fluorescence spectrophotometer (BioTek, USA). The GC376 compound was dissolved in DMSO

415 and used in a concentration range of $0.01-0.4 \mu \mathrm{M}$ to inhibit both proteases and measure their

416 kinetic parameters. Kinetic data corresponding the interaction of SARS-CoV M ${ }^{\text {pro }}$ and SARS CoV-

$4172 \mathrm{M}^{\text {pro }}$ with GC376 compound were analyzed using computer-fit calculation (Prism 4.0, GraphPad

418 Software). The slopes of the Lineweaver-Burk plots were plotted versus the inhibitor concentration

419 and the $\mathrm{K}_{\mathrm{i}}$ values were determined from the $\mathrm{x}$-axis intercept as $-\mathrm{K}_{\mathrm{i}}$.

$421 \quad$ NMR Experiments on Reversibility of Inhibitor Binding

422 The ${ }^{13} \mathrm{C}$-labelled GC376 inhibitor was synthesized according to previously documented 423 procedures, and initial HSQC NMR experiments involving only enzyme, only inhibitor, and both 424 co-incubated were prepared as previously described [13]. The sample used for the reversibility 425 experiment was prepared by subjecting a previously co-incubated sample containing both enzyme 426 and inhibitor to washing steps with buffer $\left(\mathrm{D}_{2} \mathrm{O}, 50 \mathrm{mM}\right.$ phosphate, pD 7.5 with $20 \mathrm{mM}$ DTT).

427 This involved depositing the sample in an Amicon micro-spinfilter with a $10 \mathrm{kDa}$ cutoff and 428 spinning down the sample at $6600 \mathrm{~g}$ for $15 \mathrm{~min}$. The sample was then diluted to $300 \mu \mathrm{L}$ and the 429 spin down and dilution steps were repeated once more, to a final volume of $300 \mu \mathrm{L}$. This sample 430 was then analyzed by NMR in an HSQC experiment, following protocols identical to those 431 previously described [13].

433 Reversibility and Stability of 3CL Proteases from SARS-CoV and SARS-CoV-2

434 Reversibility of 3CL protease inhibition with GC376 was determined by dialysis method. The 435 proteases were incubated with a single concentration $(20 \mu \mathrm{M})$ of the GC376 compound for 15 min 
436 at RT to allow for full inhibition. Then the enzyme-inhibitor mixture was placed in a $6-8 \mathrm{kDa}$

437 MWCO dialysis membrane (Fisher Scientific, Canada) and dialyzed against 2 L of $50 \mathrm{mM}$ Tris-

$438 \mathrm{HCl}, \mathrm{pH} 7.8,150 \mathrm{mM} \mathrm{NaCl}, 5 \%$ glycerol, $1 \mathrm{mM}$ DTT at RT. The dialysis buffer was changed every

43924 hours. Control experiments, which included dialyzing apo-proteases at the same concentration

440 in the same dialysis buffer but different beakers, were performed simultaneously. The aliquots of

441 dialyzing samples were taken out at certain time points and used for activity measurements. The

442 data was represented as a percent of initial protease activity at a zero time point.

443 The thermal stability was determined by heating $2 \mu \mathrm{M}$ solution of $\mathrm{M}^{\text {pro }} \mathrm{SARS}$ CoV or M ${ }^{\text {pro }} \mathrm{SARS}-$

444 CoV-2 in $50 \mathrm{mM}$ Tris-HCl, $\mathrm{pH} 7.8,150 \mathrm{mM} \mathrm{NaCl}, 5 \%$ glycerol, $1 \mathrm{mM}$ DTT buffer in a

445 thermostatted water-bath at various temperatures. $30 \mu 1$ protein samples were taken out at specific

446 time points and immediately incubated on ice until activity measurements were performed as

447 described above. Residual activities were expressed as relative to the maximal activity, which was

448 the activity of proteases at zero time point.

449 The enzyme inactivation over time is described by a first-order equation:

$$
\ln \left(A t / A_{0}\right)=-k t
$$

451 where $A$ represents enzyme activity at time $t, A_{0}$ is the initial activity at time zero, $k$ is the rate

452 constant $\left(\mathrm{min}^{-1}\right)$, and $t$ is time (min). Inactivation rate constants $(k d)$ were obtained from slopes of 453 semi-logarithmical plots of residual activity versus incubation time at each temperature. Calculated 454 rate constants were replotted in Arrhenius plots as natural logarithms of $k$ versus the reciprocal of 455 absolute temperature. Arrhenius law describes the temperature dependence of rate constant as

$$
\ln (k)=-E a / R T+c
$$

457 where $E a$ is the activation energy, $R$ is the universal gas constant $\left(8.31 \mathrm{~J} \mathrm{~mol}^{-1} \mathrm{~K}^{-1}\right)$, and $T$ is the 458 absolute temperature. Ea was calculated from the slope of Arrhenius plot. 
459 The half-life of proteases $\left(t_{1 / 2}\right)$, defined as time after which activity is reduced to $50 \%$ of initial

460 value [46], was determined as

$$
t_{1 / 2}=\ln (2) / k
$$

462 Another common way to present inactivation rate is as $D$ value - decimal reduction time, which is 463 the time required to reduce activity to $10 \%$ of the original value and calculated as:

$$
D=\ln (10) / k
$$

465 The activation free energy $\left(\triangle G, \mathrm{~kJ} \mathrm{~mol}^{-1}\right)$, enthalpy $\left(\Delta \mathrm{H}^{o}, \mathrm{~kJ} \mathrm{~mol}^{-1}\right)$ and entropy $\left(\Delta S^{o}, \mathrm{~kJ} \mathrm{~mol}^{-1} \mathrm{~K}^{-}\right.$

$\left.462^{1}\right)$ were determined as

$$
\Delta G=-R T \ln \left(k h / k_{B} T\right)
$$

$$
\Delta H=E a-R T
$$

$$
\Delta S=(\Delta H-\Delta G) / T
$$

470 where $h$ is the Planck constant $\left(6.626 \times 10^{-34} \mathrm{Js}\right)$ and $k_{B}$ is the Boltzmann constant $\left(1.38 \times 10^{-23} \mathrm{~J}\right.$

$\left.471 \mathrm{~K}^{-1}\right)$. Experiments were performed in duplicate.

474 For crystallization, purified SARS-CoV M $\mathrm{M}^{\text {pro }}$ and SARS-CoV-2 M $\mathrm{M}^{\text {pro }}$ were dialysed against buffer 475 containing $10 \mathrm{mM} \mathrm{NaCl}$ and $5 \mathrm{mM}$ Tris $\mathrm{HCl} \mathrm{pH} 8.0$ overnight at $4{ }^{\circ} \mathrm{C}$. Both proteins were 476 concentrated with a Millipore centrifugal filter (10 kDa MW cut-off) to a concentration of 9 $477 \mathrm{mg} / \mathrm{mL}$. Protein was incubated with 5 molar excess of inhibitor at $4{ }^{\circ} \mathrm{C}$ for $2 \mathrm{~h}$ prior to 478 crystallization. For SARS-CoV M ${ }^{\text {pro }}$, crystals were screened around previously known established 479 conditions [13] with the best crystals forming with vapour diffusion hanging drop trays at room 480 temperature at a ratio of $1: 1$ with mother liquor containing $10 \mathrm{mM} \mathrm{CaCl}_{2}, 7 \%$ PEG $8000,1 \mathrm{mM}$ 481 MES pH 6.0, 1mM DTT, 3\% ethylene glycol and 3\% DMSO (Data not shown). For SARS-CoV- 
$2 \mathrm{M}^{\text {pro }}$, the protein was subjected to the PACT crystallization screen (Molecular Dimensions,

483 USA), with hits identified in several conditions for both inhibitors. Best crystals were observed

484 with hanging drop trays at room temperature at a ratio of 1:1 with mother liquor 0.2 M Sodium

485 sulfate, $0.1 \mathrm{M}$ Bis-Tris propane $\mathrm{pH}$ 6.5, 20\% w/v PEG 3350. While the SARS-CoV-2 Mpro with

486 ligands crystallize with mother liquid containing 0.2 M Sodium chloride 0.1 M HEPES pH 7.0 20

$487 \% \mathrm{w} / \mathrm{v}$ PEG 6000. Prior to freezing, crystals were incubated with $15 \%$ glycerol as a cryoprotectant

488 for SARS-CoV-2 $\mathrm{M}^{\text {pro }}$ and $20 \%$ ethylene glycol for SARS-CoV M ${ }^{\text {pro }}$. Crystals were initially

489 screened at in-house 007 MicroMax (Rigaku Inc) with final data collection at Stanford

490 Synchrotron Radiation Lightsource SSRL, USA, beamline 12-2 with Blu-Ice using the Web-Ice

491 interface [47].

492

Diffraction Data Collection, Phase Determination, Model Building, and Refinement.

494 All diffraction data sets were collected using synchrotron radiation of wavelength $0.97946 \AA$ at 495 beamline 12-2 of Stanford Synchrotron Radiation Lightsource (SSRL) California, USA, using a 496 Dectris PILATUS 6M detector. Several data sets were collected from the crystals of SARS-CoV-

$4972 \mathrm{M}^{\text {pro }}$ free enzyme as well as with GC376 and GC373 treated. Numerous data sets were also 498 collected for SARS-CoV in the presence of GC376 and GC373. XDS2 [48] and Scala were used

499 for processing the data sets. The diffraction data set of the free SARS-CoV-2 M ${ }^{\text {pro }}$ was processed 500 at a resolution of $1.75 \AA$, in space group P21 (Supplementary Table 1). For the complex of SARS$501 \mathrm{CoV}-2 \mathrm{M}^{\text {pro }}$ with GC376 and GC373, the data set collected, was processed at a resolution of $1.9 \AA$ 502 and $2.0 \AA$ and in space group C2 (Supplementary Table 1). All three structures were determined 503 by molecular replacement with the crystal structure of the free enzyme of the SARS-CoV-2 Mpro 504 (PDB entry 6Y7M as search model, using the Phaser program from Phenix[49], version v1.18.1- 
3855). SARS-CoV M $M^{\text {pro }}$ data were also processed with XDS231 and Scala at a resolution of 2.15

$506 \AA$ and $1.90 \AA$ for GC376 and GC373, respectively, in a space group C2. Ligand Fit from Phenix

507 [50] was employed for the fitting of both inhibitors in the density of pre-calculated map from

508 Phenix refinement, using the ligand code K36. Refinement of all the structures was performed

509 with phenix.refine in Phenix software. Statistics of diffraction, data processing and model

510 refinement are given in (Supplementary Table 1). The model was inspected with Ramachandran

511 plots and all show good stereochemistry. Final models displayed using PyMOL molecular graphics

512 software (Version 2.0 Schrödinger, LLC).

513

514 Competing interests: The authors declare no competing interests.

Author contributions: J.C.V., W.V. and T.L. contributed to inhibitor synthesis. T.L. contributed

517 to FRET-substrate synthesis. E.A., M.J.v.B., J.L. and C.F. contributed to purified protein. C.F. and 518 E.A. contributed to enzyme kinetics and reversibility studies. M.J.L., H.S.Y. E.A., J.L. and M.B.K.

519 contributed to crystallization and structure determination. W. V. and R. T. M. contributed to 520 labelled NMR studies. M.J.L wrote the initial draft. All authors read and approved the manuscript.

522 Acknowledgements. We would like to thank the staff at the Stanford Synchrotron Light Source, 523 in particular Dr. Silvia Russi and Lisa Dunn. M.J.L, J.C.V and D.L.T. acknowledge funding from 524 CIHR and NSERC (COVID-19 SOF-549297-2019). D.L.T acknowledges support from Li Ka 525 Shing Institute of Virology and the GSK Chair in Virology. W.V. was supported by an Alberta 526 Innovates Graduate Scholarship and an Alberta Graduate Excellence Scholarship. T.L. was 527 supported by a CIHR Vanier Scholarship. 
Use of the Stanford Synchrotron Radiation Lightsource, SLAC National Accelerator Laboratory, is supported by the U.S. Department of Energy, Office of Science, Office of Basic Energy Sciences under Contract No. DE-AC02-76SF00515. The SSRL Structural Molecular Biology Program is supported by the DOE Office of Biological and Environmental Research, and by the National

533 Institutes of Health, National Institute of General Medical Sciences (P41GM103393). The 534 contents of this publication are solely the responsibility of the authors and do not necessarily 535 represent the official views of NIGMS or NIH.

\section{References}

1. Wu F, Zhao S, Yu B, Chen YM, Wang W, et al. (2020) A new coronavirus associated with human respiratory disease in China. Nature 579: 265-269.

2. Lu R, Zhao X, Li J, Niu P, Yang B, et al. (2020) Genomic characterisation and epidemiology of 2019 novel coronavirus: implications for virus origins and receptor binding. Lancet 395: 565-574.

3. Wu A, Peng Y, Huang B, Ding X, Wang X, et al. (2020) Genome Composition and Divergence of the Novel Coronavirus (2019-nCoV) Originating in China. Cell Host Microbe 27: 325328.

4. Gorbalenya AE, Baker SC (2020) The species Severe acute respiratory syndrome-related coronavirus: classifying 2019-nCoV and naming it SARS-CoV-2. Nat Microbiol 5: 536544.

5. Hilgenfeld R (2014) From SARS to MERS: crystallographic studies on coronaviral proteases enable antiviral drug design. FEBS J 281: 4085-4096.

6. Grum-Tokars V, Ratia K, Begaye A, Baker SC, Mesecar AD (2008) Evaluating the 3C-like protease activity of SARS-Coronavirus: recommendations for standardized assays for drug discovery. Virus Res 133: 63-73.

7. Muramatsu T, Takemoto C, Kim YT, Wang H, Nishii W, et al. (2016) SARS-CoV 3CL protease cleaves its C-terminal autoprocessing site by novel subsite cooperativity. Proc Natl Acad Sci U S A 113: 12997-13002.

8. Rut W, Groborz K, Zhang L, Sun X, Zmudzinski M, et al. (2021) SARS-CoV-2 M(pro) inhibitors and activity-based probes for patient-sample imaging. Nat Chem Biol 17: 222228. 
9. Kneller DW, Galanie S, Phillips G, O'Neill HM, Coates L, et al. (2020) Malleability of the SARS-CoV-2 3CL M(pro) Active-Site Cavity Facilitates Binding of Clinical Antivirals. Structure 28: 1313-1320 e1313.

10. Zhang L, Lin D, Sun X, Curth U, Drosten C, et al. (2020) Crystal structure of SARS-CoV-2 main protease provides a basis for design of improved alpha-ketoamide inhibitors. Science 368: 409-412.

11. Jin Z, Du X, Xu Y, Deng Y, Liu M, et al. (2020) Structure of M(pro) from SARS-CoV-2 and discovery of its inhibitors. Nature 582: 289-293.

12. Kumar Y, Singh H, Patel CN (2020) In silico prediction of potential inhibitors for the main protease of SARS-CoV-2 using molecular docking and dynamics simulation based drugrepurposing. J Infect Public Health 13: 1210-1223.

13. Vuong W, Khan MB, Fischer C, Arutyunova E, Lamer T, et al. (2020) Feline coronavirus drug inhibits the main protease of SARS-CoV-2 and blocks virus replication. Nat Commun 11: 4282.

14. Anderson J, Schiffer C, Lee SK, Swanstrom R (2009) Viral Protease Inhibitors. In: Kräusslich $\mathrm{H}$, Bartenschlager R, editors. Antiviral Strategies: Springer. pp. 1-24.

15. Ma C, Sacco MD, Hurst B, Townsend JA, Hu Y, et al. (2020) Boceprevir, GC-376, and calpain inhibitors II, XII inhibit SARS-CoV-2 viral replication by targeting the viral main protease. Cell Res 30: 678-692.

16. Kim Y, Lovell S, Tiew KC, Mandadapu SR, Alliston KR, et al. (2012) Broad-spectrum antivirals against $3 \mathrm{C}$ or $3 \mathrm{C}$-like proteases of picornaviruses, noroviruses, and coronaviruses. J Virol 86: 11754-11762.

17. Kim Y, Liu H, Galasiti Kankanamalage AC, Weerasekara S, Hua DH, et al. (2016) Reversal of the Progression of Fatal Coronavirus Infection in Cats by a Broad-Spectrum Coronavirus Protease Inhibitor. PLoS Pathog 12: e1005531.

18. Rathnayake AD, Zheng J, Kim Y, Perera KD, Mackin S, et al. (2020) 3C-like protease inhibitors block coronavirus replication in vitro and improve survival in MERS-CoVinfected mice. Sci Transl Med 12.

19. Galasiti Kankanamalage AC, Kim Y, Weerawarna PM, Uy RA, Damalanka VC, et al. (2015) Structure-guided design and optimization of dipeptidyl inhibitors of norovirus $3 \mathrm{CL}$ protease. Structure-activity relationships and biochemical, X-ray crystallographic, cellbased, and in vivo studies. J Med Chem 58: 3144-3155.

20. Kim Y, Shivanna V, Narayanan S, Prior AM, Weerasekara S, et al. (2015) Broad-spectrum inhibitors against $3 \mathrm{C}$-like proteases of feline coronaviruses and feline caliciviruses. J Virol 89: 4942-4950.

21. Galasiti Kankanamalage AC, Kim Y, Damalanka VC, Rathnayake AD, Fehr AR, et al. (2018) Structure-guided design of potent and permeable inhibitors of MERS coronavirus 3CL protease that utilize a piperidine moiety as a novel design element. Eur J Med Chem 150: 334-346.

22. Perera KD, Galasiti Kankanamalage AC, Rathnayake AD, Honeyfield A, Groutas W, et al. (2018) Protease inhibitors broadly effective against feline, ferret and mink coronaviruses. Antiviral Res 160: 79-86.

23. Chang KO, Kim Y, Lovell S, Rathnayake AD, Groutas WC (2019) Antiviral Drug Discovery: Norovirus Proteases and Development of Inhibitors. Viruses 11.

24. Drag M, Salvesen GS (2010) Emerging principles in protease-based drug discovery. Nat Rev Drug Discov 9: 690-701. 
25. Hoffman RL, Kania RS, Brothers MA, Davies JF, Ferre RA, et al. (2020) Discovery of KetoneBased Covalent Inhibitors of Coronavirus 3CL Proteases for the Potential Therapeutic Treatment of COVID-19. J Med Chem 63: 12725-12747.

26. Turk B (2006) Targeting proteases: successes, failures and future prospects. Nat Rev Drug Discov 5: 785-799.

27. Sinclair JF, Ziegler MM, Baldwin TO (1994) Kinetic partitioning during protein folding yields multiple native states. Nat Struct Biol 1: 320-326.

28. Chen J, Wharton SA, Weissenhorn W, Calder LJ, Hughson FM, et al. (1995) A soluble domain of the membrane-anchoring chain of influenza virus hemagglutinin (HA2) folds in Escherichia coli into the low-pH-induced conformation. Proc Natl Acad Sci U S A 92: 12205-12209.

29. Lai Z, McCulloch J, Lashuel HA, Kelly JW (1997) Guanidine hydrochloride-induced denaturation and refolding of transthyretin exhibits a marked hysteresis: equilibria with high kinetic barriers. Biochemistry 36: 10230-10239.

30. Barrientos LG, Louis JM, Botos I, Mori T, Han Z, et al. (2002) The domain-swapped dimer of cyanovirin-N is in a metastable folded state: reconciliation of X-ray and NMR structures. Structure 10: 673-686.

31. Fasshauer D, Antonin W, Subramaniam V, Jahn R (2002) SNARE assembly and disassembly exhibit a pronounced hysteresis. Nat Struct Biol 9: 144-151.

32. Malcolm BA, Lowe C, Shechosky S, McKay RT, Yang CC, et al. (1995) Peptide aldehyde inhibitors of hepatitis A virus 3C proteinase. Biochemistry 34: 8172-8179.

33. Maseko S, Padayachee E, Maphumulo S, Govender T, Sayed Y, et al. (2019) Kinetic and thermodynamic characterisation of HIV-protease inhibitors against E35D upward arrowG upward arrowS mutant in the South African HIV-1 subtype C protease. J Enzyme Inhib Med Chem 34: 1451-1456.

34. Gouzi H, Depagne C, Coradin T (2012) Kinetics and thermodynamics of the thermal inactivation of polyphenol oxidase in an aqueous extract from Agaricus bisporus. J Agric Food Chem 60: 500-506.

35. Dogan N, Tari C (2008) Characterization of three-phase partitioned exo-polygalacturonase from Aspergillus sojae with unique properties. Biochemical Engineering Journal 39: 4350 .

36. Hu T, Zhang Y, Li L, Wang K, Chen S, et al. (2009) Two adjacent mutations on the dimer interface of SARS coronavirus 3C-like protease cause different conformational changes in crystal structure. Virology 388: 324-334.

37. Chen S, Zhang J, Hu T, Chen K, Jiang H, et al. (2008) Residues on the dimer interface of SARS coronavirus $3 \mathrm{C}$-like protease: dimer stability characterization and enzyme catalytic activity analysis. J Biochem 143: 525-536.

38. Xue X, Yang H, Shen W, Zhao Q, Li J, et al. (2007) Production of authentic SARS-CoV $\mathrm{M}$ (pro) with enhanced activity: application as a novel tag-cleavage endopeptidase for protein overproduction. J Mol Biol 366: 965-975.

39. Lee J, Worrall LJ, Vuckovic M, Rosell FI, Gentile F, et al. (2020) Crystallographic structure of wild-type SARS-CoV-2 main protease acyl-enzyme intermediate with physiological Cterminal autoprocessing site. Nat Commun 11: 5877.

40. Thorarensen A, Balbo P, Banker ME, Czerwinski RM, Kuhn M, et al. (2021) The advantages of describing covalent inhibitor in vitro potencies by IC50 at a fixed time point. IC50 
determination of covalent inhibitors provides meaningful data to medicinal chemistry for SAR optimization. Bioorg Med Chem 29: 115865.

41. Howe AY, Venkatraman S (2013) The Discovery and Development of Boceprevir: A Novel, First-generation Inhibitor of the Hepatitis C Virus NS3/4A Serine Protease. J Clin Transl Hepatol 1: 22-32.

42. Soumana DI, Kurt Yilmaz N, Ali A, Prachanronarong KL, Schiffer CA (2016) Molecular and Dynamic Mechanism Underlying Drug Resistance in Genotype 3 Hepatitis C NS3/4A Protease. J Am Chem Soc 138: 11850-11859.

43. Anand K, Ziebuhr J, Wadhwani P, Mesters JR, Hilgenfeld R (2003) Coronavirus main proteinase (3CLpro) structure: basis for design of anti-SARS drugs. Science 300: 17631767.

44. Shi JH, Song JX (2006) The catalysis of the SARS 3C-like protease is under extensive regulation by its extra domain. Febs Journal 273: 1035-1045.

45. Ye G, Wang X, Tong X, Shi Y, Fu ZF, et al. (2020) Structural Basis for Inhibiting Porcine Epidemic Diarrhea Virus Replication with the 3C-Like Protease Inhibitor GC376. Viruses 12.

46. Sadana A (1991) Biocatalysis: fundamentals of enzyme deactivation kinetics. Englewood Cliffs, N.J. : Prentice Hall

47. Gonzalez A, Moorhead P, McPhillips SE, Song J, Sharp K, et al. (2008) Web-Ice: integrated data collection and analysis for macromolecular crystallography. Journal of Applied Crystallography 41: 176-184.

48. Kabsch W (2010) Xds. Acta Crystallogr D Biol Crystallogr 66: 125-132.

49. Liebschner D, Afonine PV, Baker ML, Bunkoczi G, Chen VB, et al. (2019) Macromolecular structure determination using X-rays, neutrons and electrons: recent developments in Phenix. Acta Crystallogr D Struct Biol 75: 861-877.

50. Adams PD, Afonine PV, Bunkoczi G, Chen VB, Davis IW, et al. (2010) PHENIX: a comprehensive Python-based system for macromolecular structure solution. Acta Crystallogr D Biol Crystallogr 66: 213-221. 


\begin{tabular}{|l|l|l|}
\hline Protease & $\mathbf{I C}_{\mathbf{5 0}}, \boldsymbol{\mu} \mathbf{M}$ & Calculated Ki, $\boldsymbol{\mu M}$ \\
\hline SARS-CoV M & \\
& $0.05 \pm 0.01$ & 0.02 \\
\hline SARS-CoV-2 M & & \\
\hline
\end{tabular}

Table 1. Comparison of $\mathrm{IC}_{50}$ and $\mathrm{K}_{\mathrm{i}}$ values between $\mathrm{SARS}-\mathrm{CoV} \mathrm{M}^{\text {pro }}$ and SARS-CoV-2 $\mathrm{M}^{\text {pro }}$ with

684 compound GC376. Data are presented as mean $\pm \mathrm{SEM}, n=3$.

A

686

687

688

689

\begin{tabular}{|c|c|c|c|c|c|c|c|c|}
\hline $\mathbf{t}\left({ }^{\circ} \mathbf{C}\right)$ & $\left.\mathbf{k d ~} \mathbf{m i n}^{-\mathbf{1}}\right)$ & $\mathbf{t}_{\mathbf{1} / \mathbf{2}} \mathbf{( \mathbf { m i n } )}$ & $\mathbf{t}_{\mathbf{1} / \mathbf{2}}(\mathbf{h})$ & $\mathbf{D t} \mathbf{( \mathbf { m i n } )}$ & $\mathbf{D t}(\mathbf{h})$ & $\mathbf{\Delta H}(\mathbf{k J} / \mathbf{m o l})$ & $\Delta \mathbf{G}(\mathbf{k J} / \mathbf{m o l})$ & $\Delta \mathbf{~ S}\left(\mathbf{k J} / \mathbf{m o l}{ }^{*} \mathbf{K}\right)$ \\
\hline $\mathbf{2 4}$ & 0.0003 & 2310.5 & 38.5 & 7675.3 & 127.9 & 13.9 & 82.6 & -0.23 \\
\hline $\mathbf{3 7}$ & 0.0004 & 1732.9 & 28.8 & 5756.5 & 95.9 & 240.9 & 85.6 & 0.50 \\
\hline $\mathbf{4 5}$ & 0.0011 & 630.1 & 10.5 & 2093.3 & 34.9 & 240.9 & 85.2 & 0.48 \\
\hline $\mathbf{5 5}$ & 0.318 & 2.2 & 0.04 & 7.2 & 0.121 & 240.8 & 72.5 & 0.52 \\
\hline $\mathbf{7 0}$ & 1.67 & 0.4 & 0.007 & 1.4 & 0.023 & 240.6 & 71.2 & 0.52 \\
\hline
\end{tabular}

690

691

692

693

\begin{tabular}{|c|c|c|c|c|c|c|c|c|}
\hline $\mathbf{t}\left({ }^{\circ} \mathbf{C}\right)$ & $\mathbf{k d ~}\left(\mathbf{m i n}^{-1}\right)$ & $\mathbf{t}_{\mathbf{1} / 2}(\mathbf{m i n})$ & $\mathbf{t}_{\mathbf{1} / 2}(\mathbf{h})$ & $\mathbf{D t}(\mathbf{m i n})$ & $\mathbf{D t}(\mathbf{h})$ & $\mathbf{\Delta H}(\mathbf{k J} / \mathbf{m o l})$ & $\Delta \mathbf{G}(\mathbf{k J} / \mathbf{m o l})$ & $\Delta \mathbf{~ S}\left(\mathbf{k J} / \mathbf{m o l}^{*} \mathbf{K}\right)$ \\
\hline $\mathbf{2 4}$ & 0.0002 & 3465.7 & 57.7 & 11512.9 & 191.8 & 38.9 & 83.6 & -0.15 \\
\hline $\mathbf{3 7}$ & 0.0004 & 1732.8 & 28.8 & 5756.4 & 95.9 & 176.5 & 85.6 & 0.29 \\
\hline $\mathbf{4 5}$ & 0.001 & 693.1 & 11.5 & 2302.5 & 38.3 & 176.4 & 85.4 & 0.28 \\
\hline $\mathbf{5 5}$ & 0.23 & 3 & 0.05 & 10.01 & 0.2 & 176.4 & 73.4 & 0.31 \\
\hline $\mathbf{7 0}$ & 1.2 & 0.6 & 0.009 & 1.9 & 0.03 & 176.2 & 72.2 & 0.32 \\
\hline
\end{tabular}

Table 2. Thermodynamic parameters for the thermal inactivation of (A) SARS-CoV $M^{\text {pro }}$ and (B)

699 proteases (i.e., the time after which activity is reduced to one-half of the initial value), $D t$, decimal

700 reduction time, the time required to reduce the enzymatic activity to $10 \%$ of its original value, $\Delta G$,

701 activation free energy barrier, $\Delta H$, activation enthalpy, $\Delta S$ activation entropy of thermal

702 denaturation. 


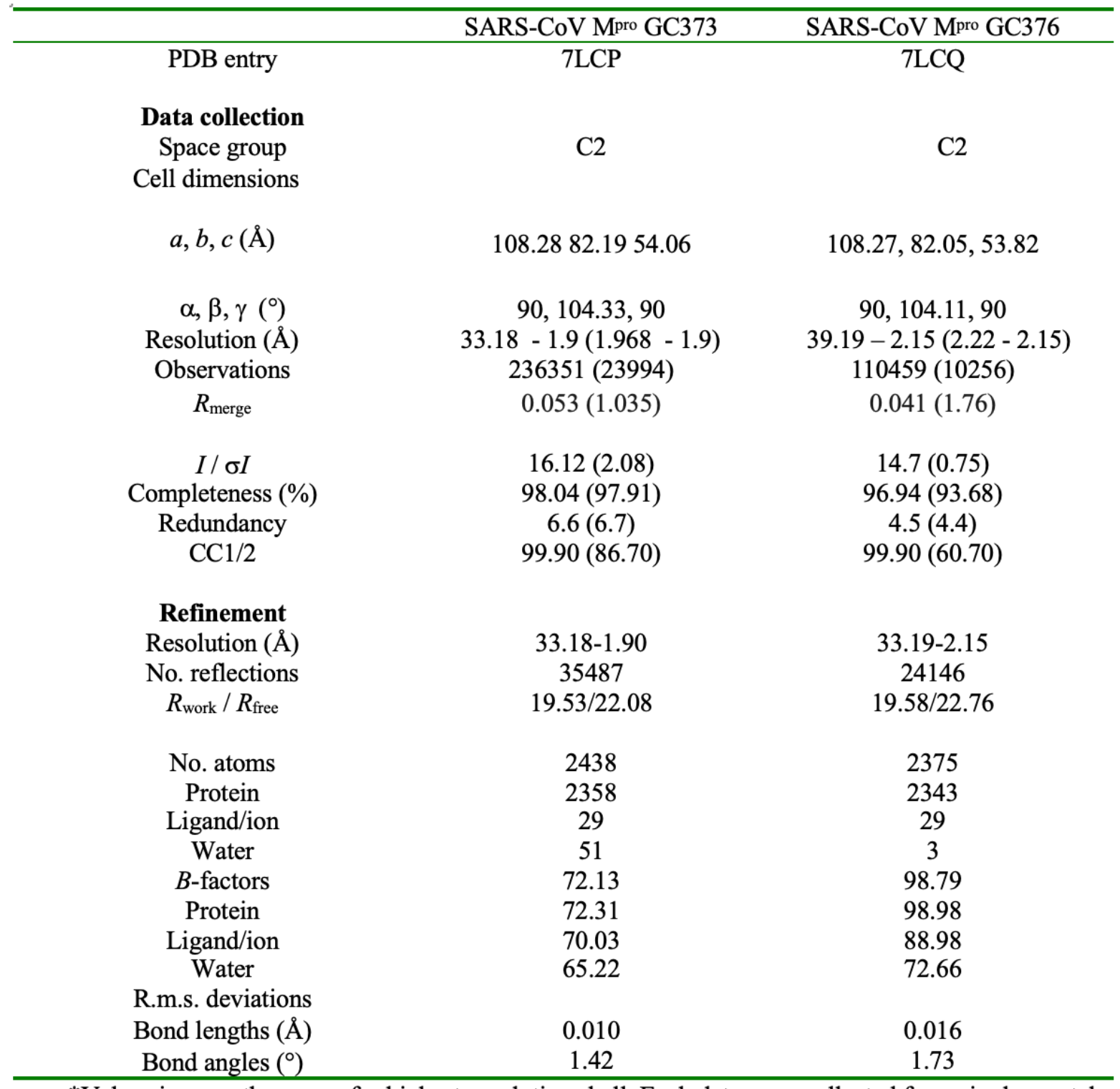

706 Table 3. Data collection and refinement statistics (molecular replacement) for SARS-CoV Mpro

707 with drug GC373 and prodrug GC376. 

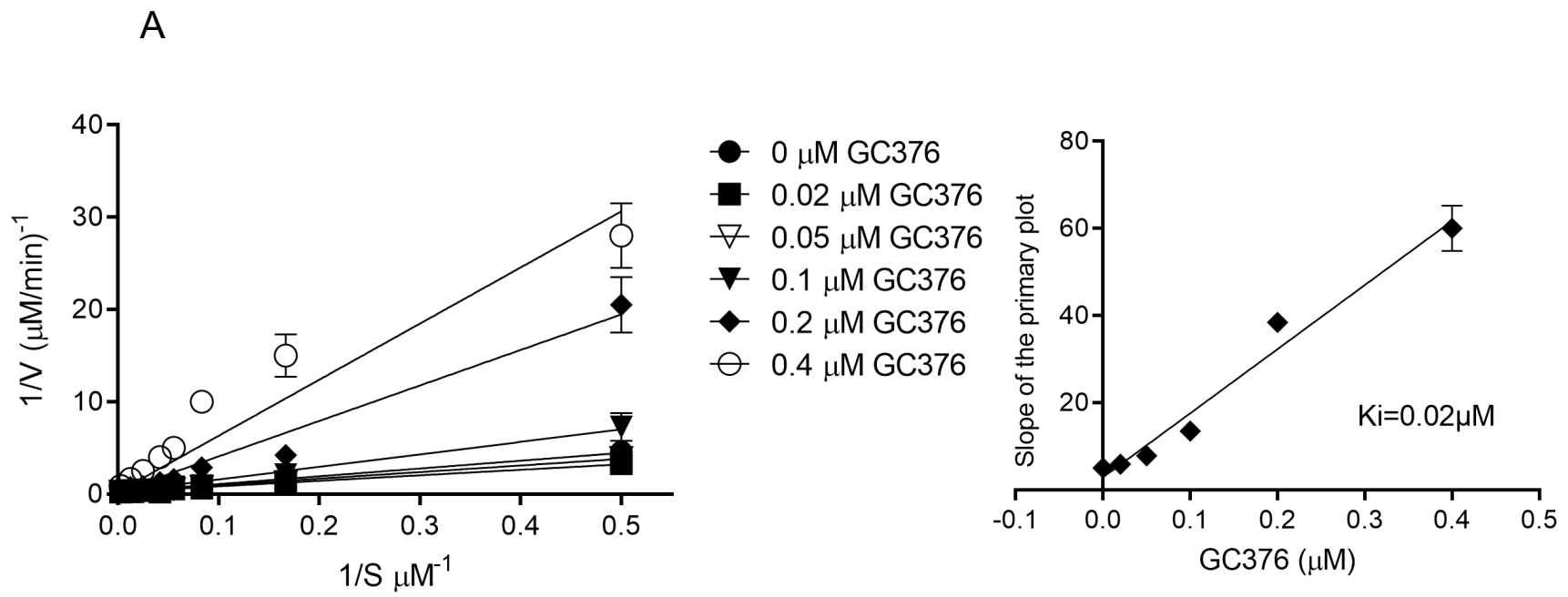

B
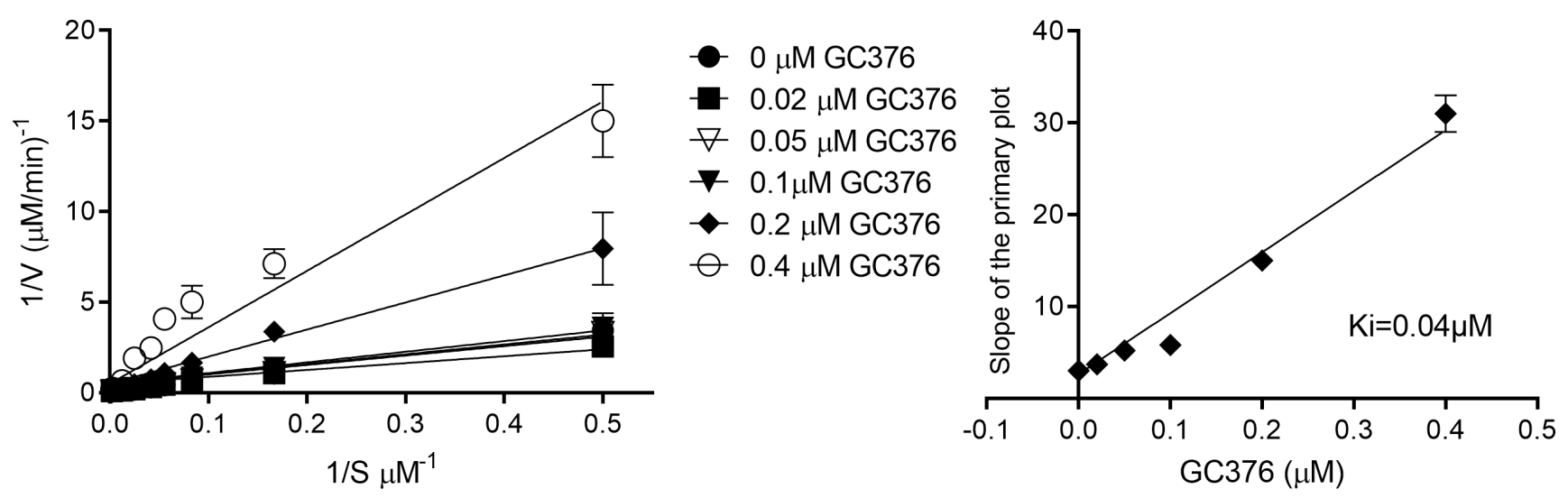

Fig 1. Determination of $K_{i}$ values of GC376 for SARS-CoV $M^{\text {pro }}$ and SARS-CoV-2 $M^{\text {pro }}$. Lineweaver-Burk plots (left) and the secondary plots of competitive inhibition (right) of SARS$\mathrm{CoV} \mathrm{M}^{\text {pro }}(\mathrm{A})$ and SARS-CoV-2 $\mathrm{M}^{\text {pro }}(\mathrm{B})$ by GC376. Data are presented as mean $\pm \mathrm{SEM}, n=3$ 
A<smiles>[2H][15NH+]([O-])C(CNC(=O)[C@H](CC(C)C)NC(=O)OCc1ccccc1)CC1CCNC1=O</smiles>

B
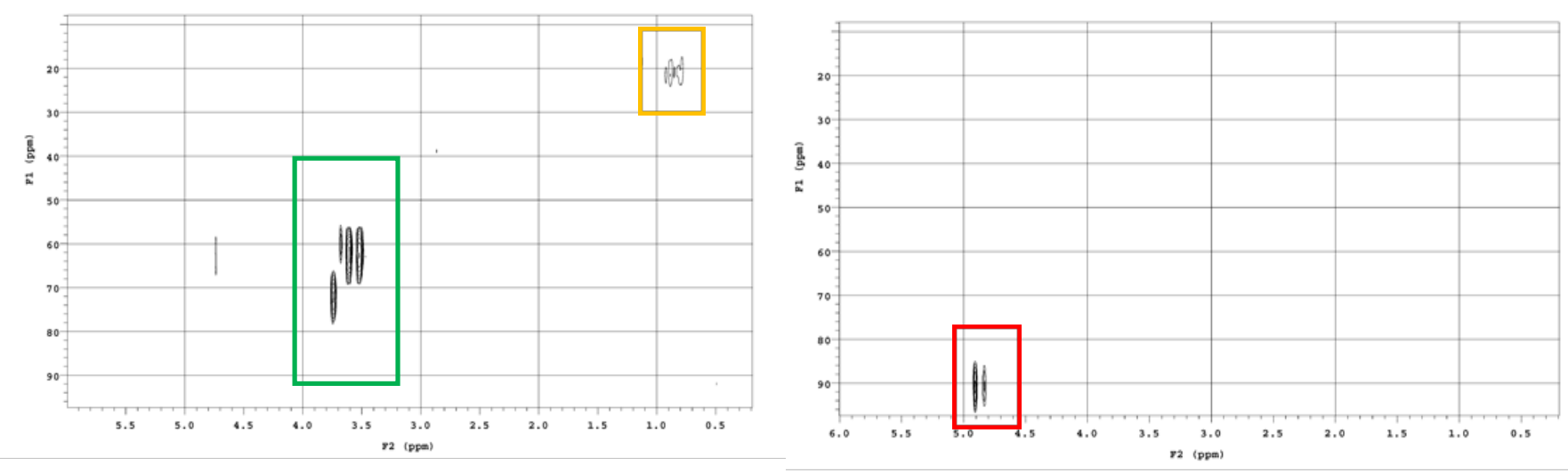

D
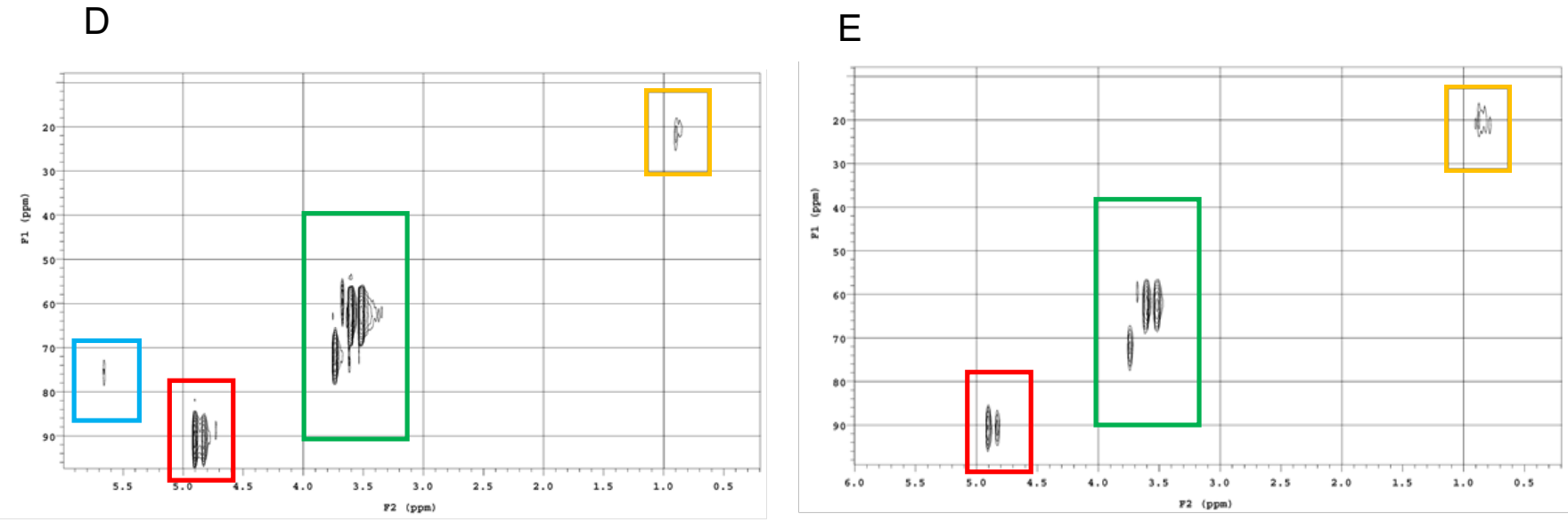

Figure 2: HSQC NMR experiments examining reversibility of GC376 binding. (A) Structure of ${ }^{13}$ C-labelled GC373. (B) HSQC spectra of SARS-CoV-2 M $\mathrm{M}^{\text {pro }}$ in deuterated buffer. (C) HSQC spectra of ${ }^{13} \mathrm{C}$-labelled GC373. (D) Co-incubation of SARS-CoV-2 $\mathrm{M}^{\text {pro }}$ with ${ }^{13} \mathrm{C}$-labelled GC373. (E) Co-incubated sample after washing step with buffer. Boxes: Blue = bound inhibitor; Red = free inhibitor; Green = DTT (from buffer); Orange = SARS-CoV-2 M ${ }^{\text {pro }}$. 
A

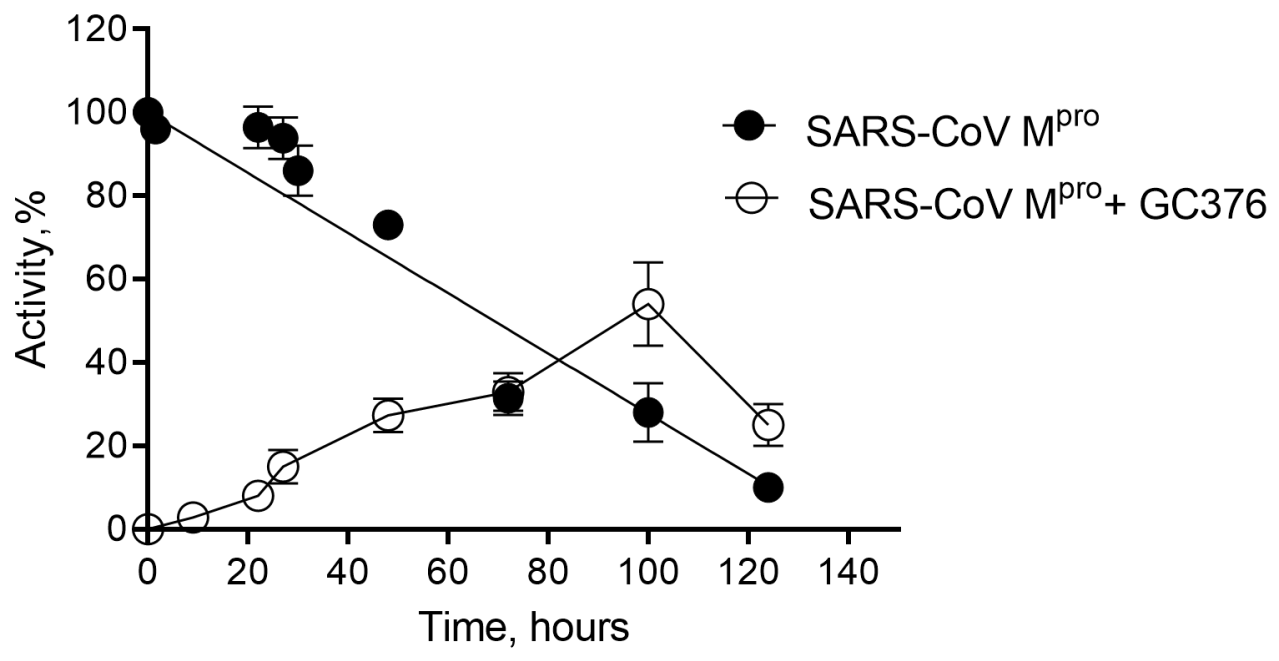

B

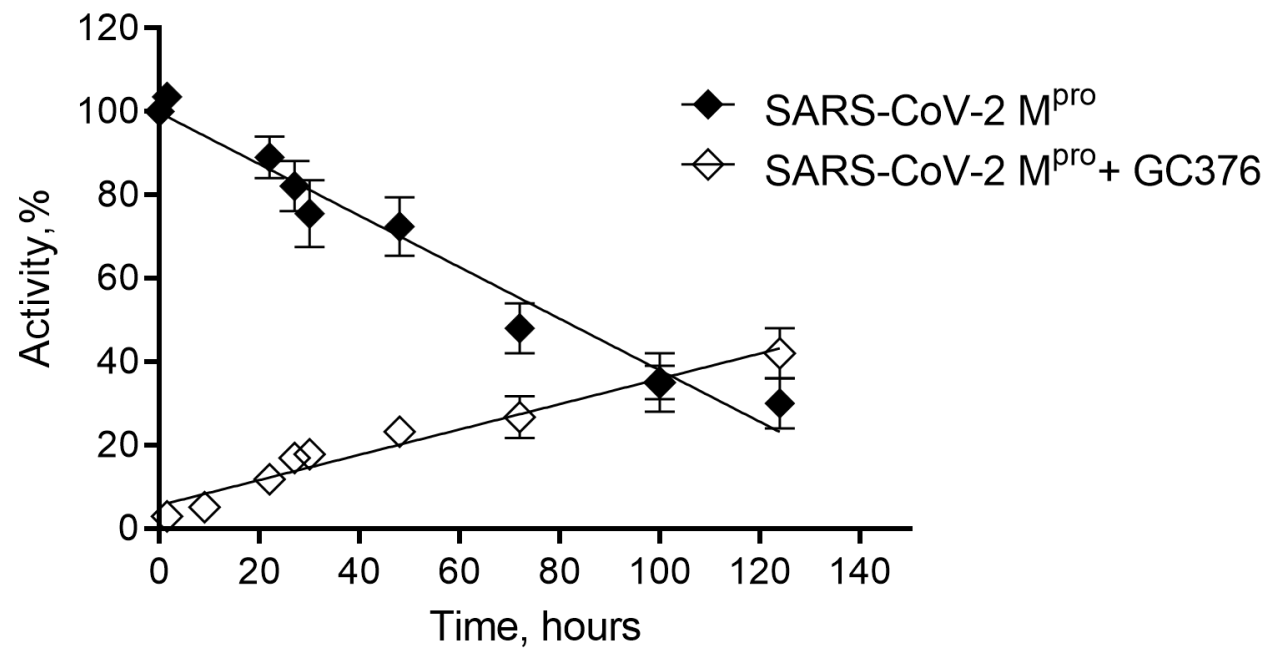

Fig 3. Reversibility of GC376 with SARS-CoV $M^{\text {pro }}$ and SARS-CoV-2 $M^{\text {pro }}$. The dependence of activity of SARS-CoV M $\mathrm{M}^{\text {pro }}$ (A) and SARS-CoV-2 $\mathrm{M}^{\text {pro }}(\mathrm{B})$ incubated alone and with the bound GC376 compound on time. Data are presented as mean $\pm \mathrm{SEM}, n=3$ 


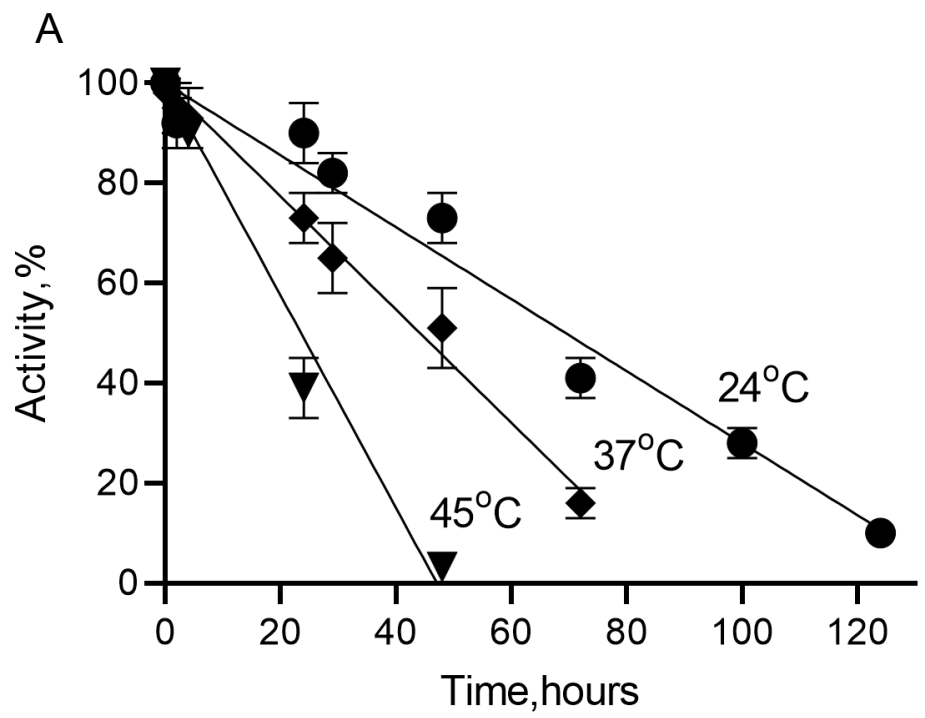

B

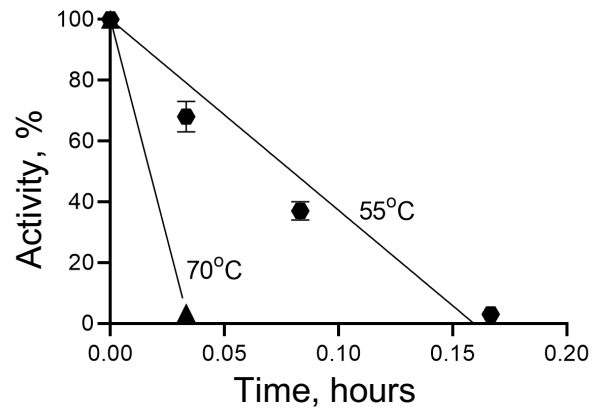

C

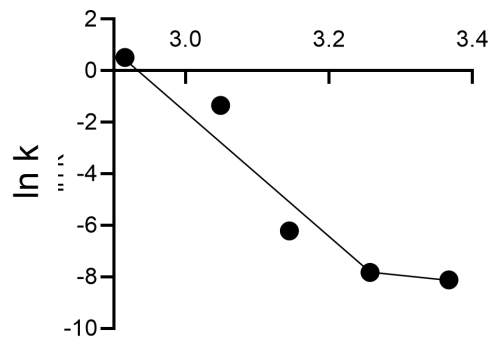

$1000 / \mathrm{T}, \mathrm{K}^{-1}$

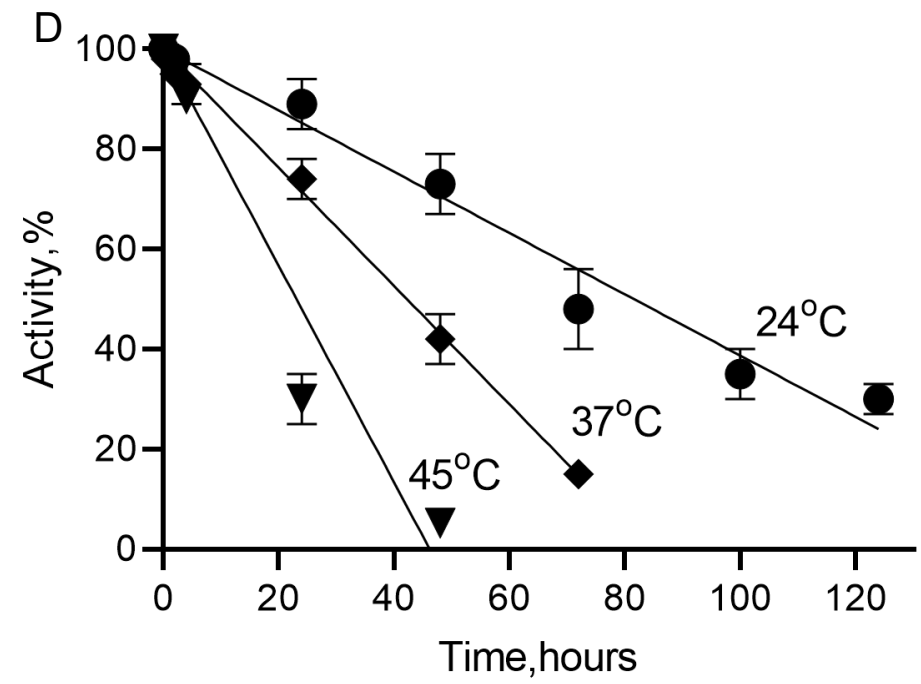

$E$

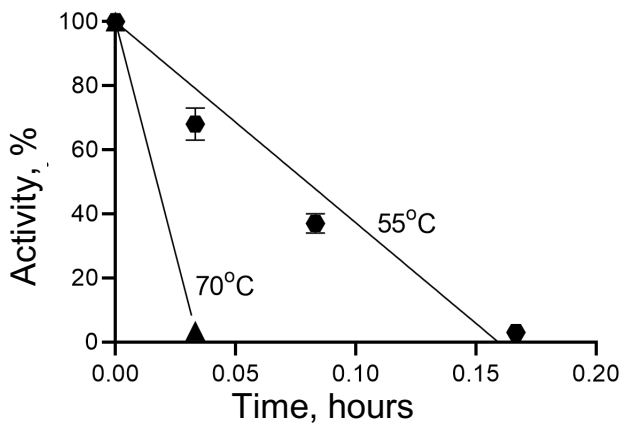

$\mathrm{F}$

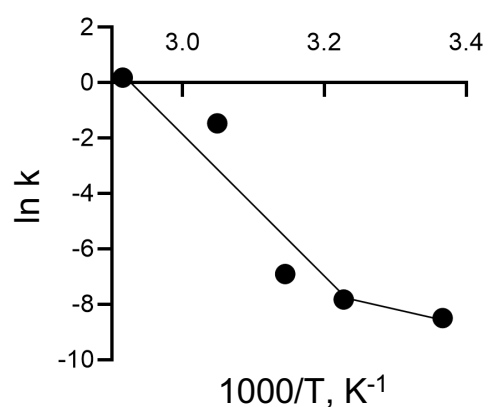

Fig 4. Thermal stability study of SARS-CoV $M^{\text {pro }}$ and SARS-CoV-2 $M^{\text {pro. }}$. Time course of residual activities of SARS-CoV $\mathrm{M}^{\text {pro }}$ and SARS-CoV-2 $\mathrm{M}^{\text {pro }}$ in temperature ranges of $24-45^{\circ} \mathrm{C}$ (A and $\mathrm{D}$, respectively) and in $55-70^{\circ} \mathrm{C}$ (B and E) and Arrhenius plots for SARS-CoV M ${ }^{\text {pro }}$ and SARS-CoV-2 $\mathrm{M}^{\text {pro }}$ (C and F, respectively). Data are presented as mean $\pm \mathrm{SEM}, n=2$ 
A

Dimerization domain Chymotrypsin-like domain

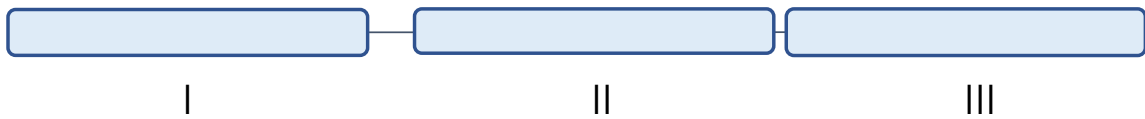

B

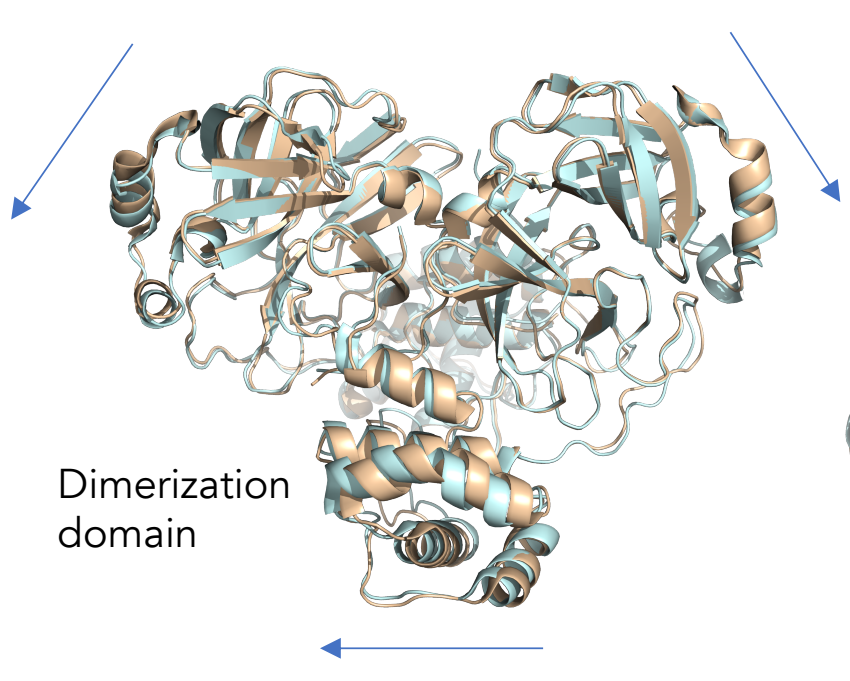

D

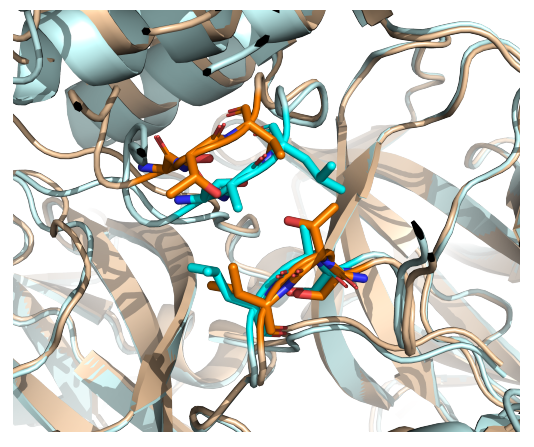

$E$

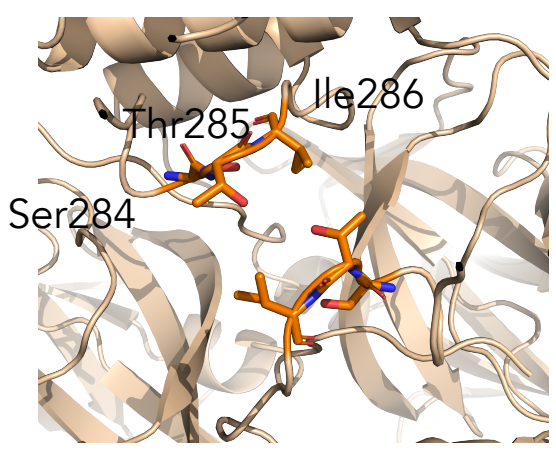

C

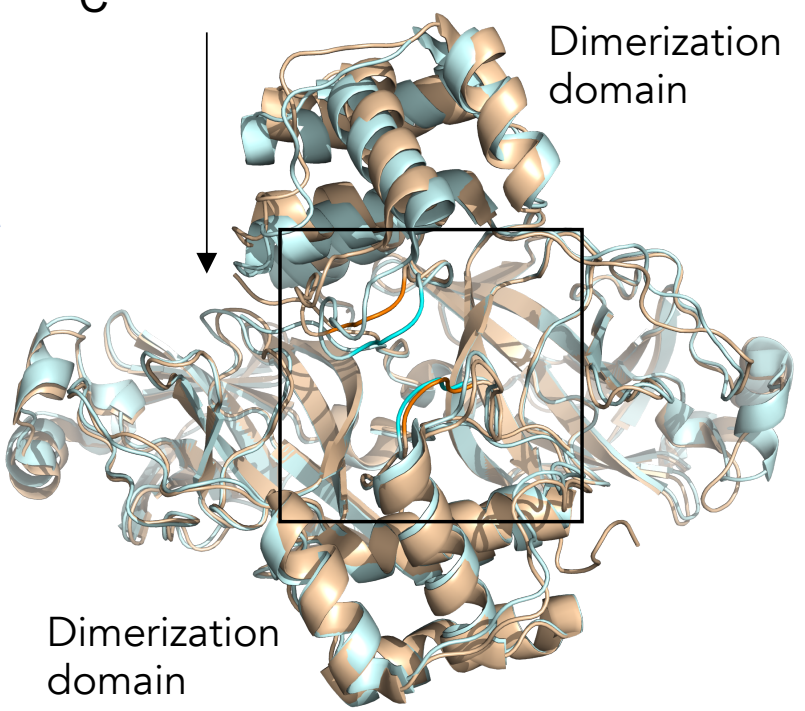

$\mathrm{F}$

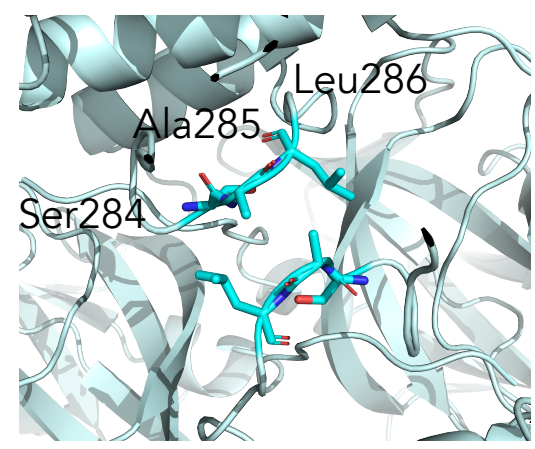

Fig 5. Differences observed between SARS-CoV and SARS-CoV-2 $M^{\text {pro }}$ structures. (A) Overall domain organization of $\mathrm{M}^{\mathrm{pro}}$. (B) Overlay of SARS-CoV $\mathrm{M}^{\text {pro }}$ (2DUC.pdb) and SARSCoV-2 M ${ }^{\text {pro }}$ (6WTM.pdb) structures reveals small global shifts. (C) The largest structural change is the closer distance between the dimer interface of $\mathrm{M}^{\text {pro }}$ in SARS-CoV-2 compared to SARSCoV. (D) A close examination of the dimerization loop in both SARS-CoV $\mathrm{M}^{\text {pro }}$ (orange) and SARS-CoV-2 $\mathrm{M}^{\text {pro }}$ (blue). (E) In SARS-CoV $\mathrm{M}^{\text {pro }}$ a Thr285 in the STI sequence at the dimer interface participates in dimerization via hydrophobic interactions while the $\mathrm{M}^{\text {pro }}$ in SARS-CoV2 (F) has an alanine in a SAL motif resulting in a zippered interdigitation of the hydrophobic residues and closer association of the dimerization domains. 


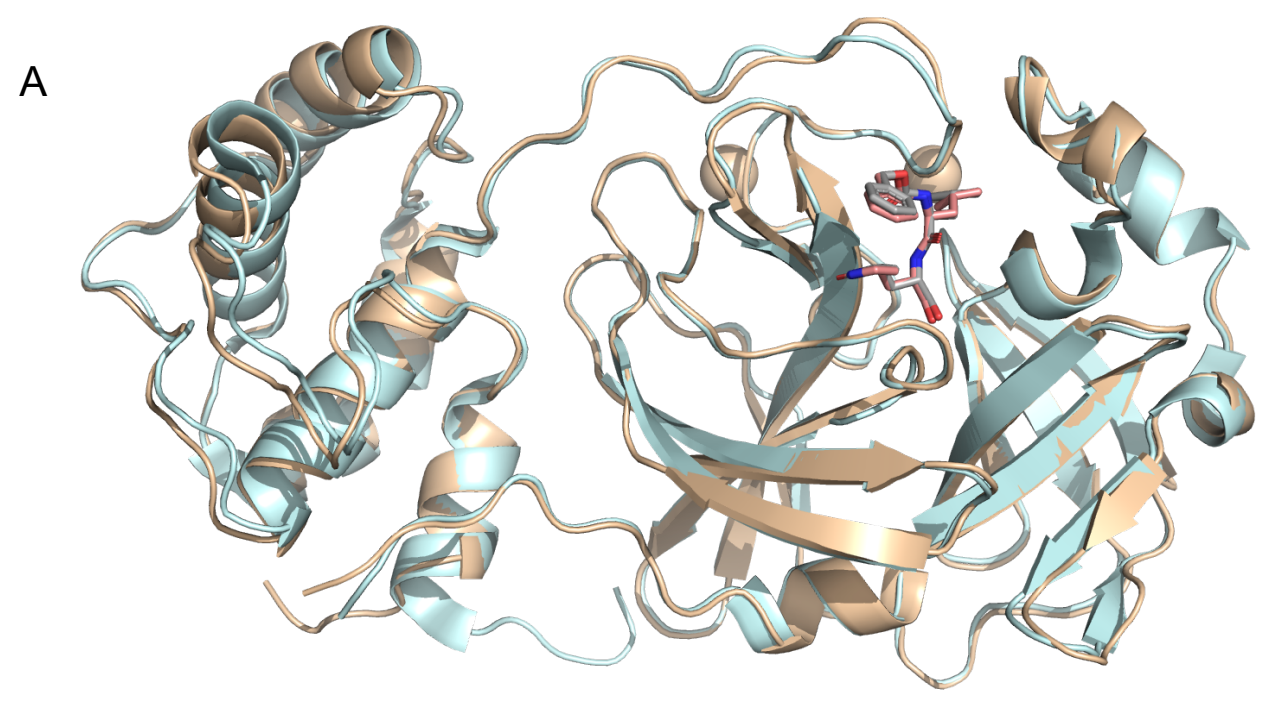

B

C
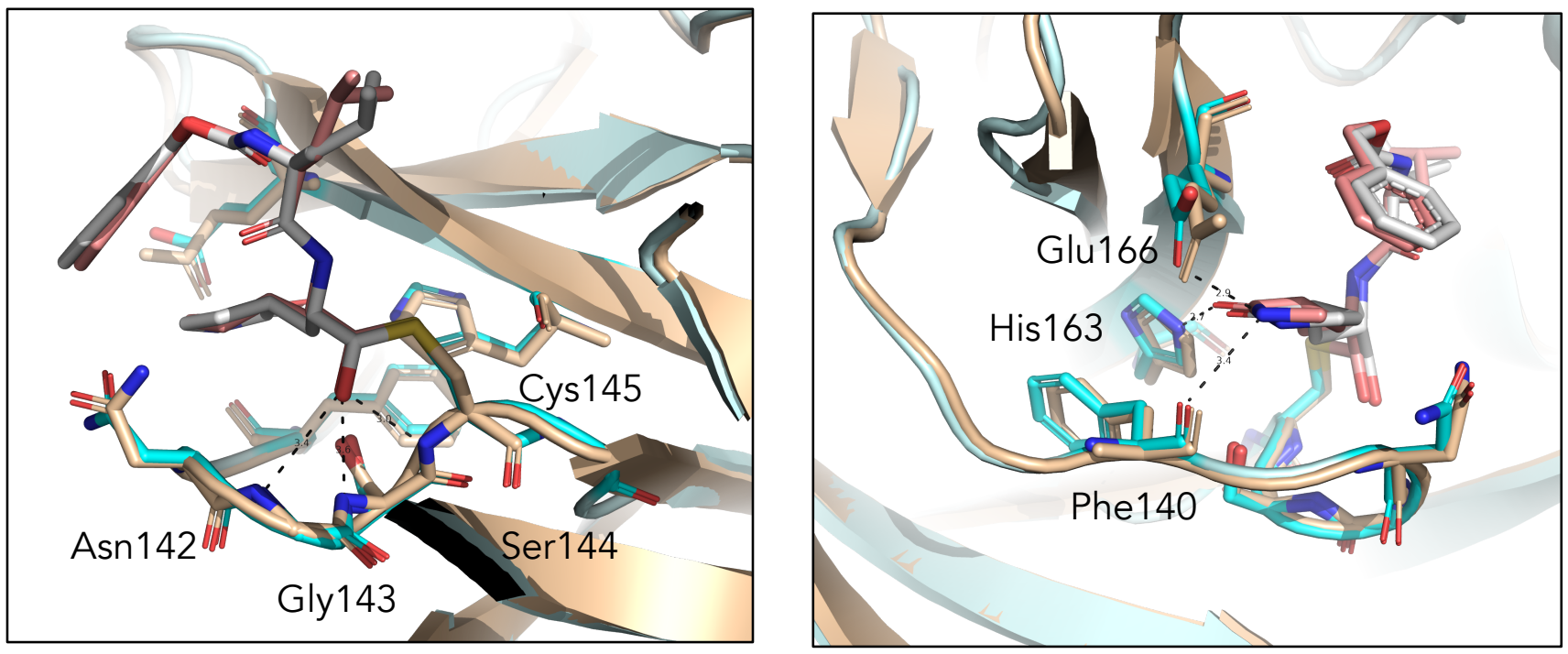

Figure 6. Comparison of SARS-CoV and SARS-CoV-2 $M^{\text {pro }}$ structures with GC373 drug. (A). Overall 3-dimensional structures show similarities between SARS-CoV M ${ }^{\text {pro }}$ (wheat) and SARS CoV-2 $\mathrm{M}^{\text {pro }}$ (cyan) with an RMSD of $0.6 \AA$. GC373 binds covalently with the catalytic Cys145 of the M $\mathrm{M}^{\text {pro }}$ of both SARS-CoV (7LCP.pdb) and SARS-CoV-2 (6TWK.pdb) and shows (B) similar oxyanion hole coordination by Ser144, Gly143 and Asn142 and (C) drug adduct coordination with side chains of His163, Glu166 and backbone of Phe140. 


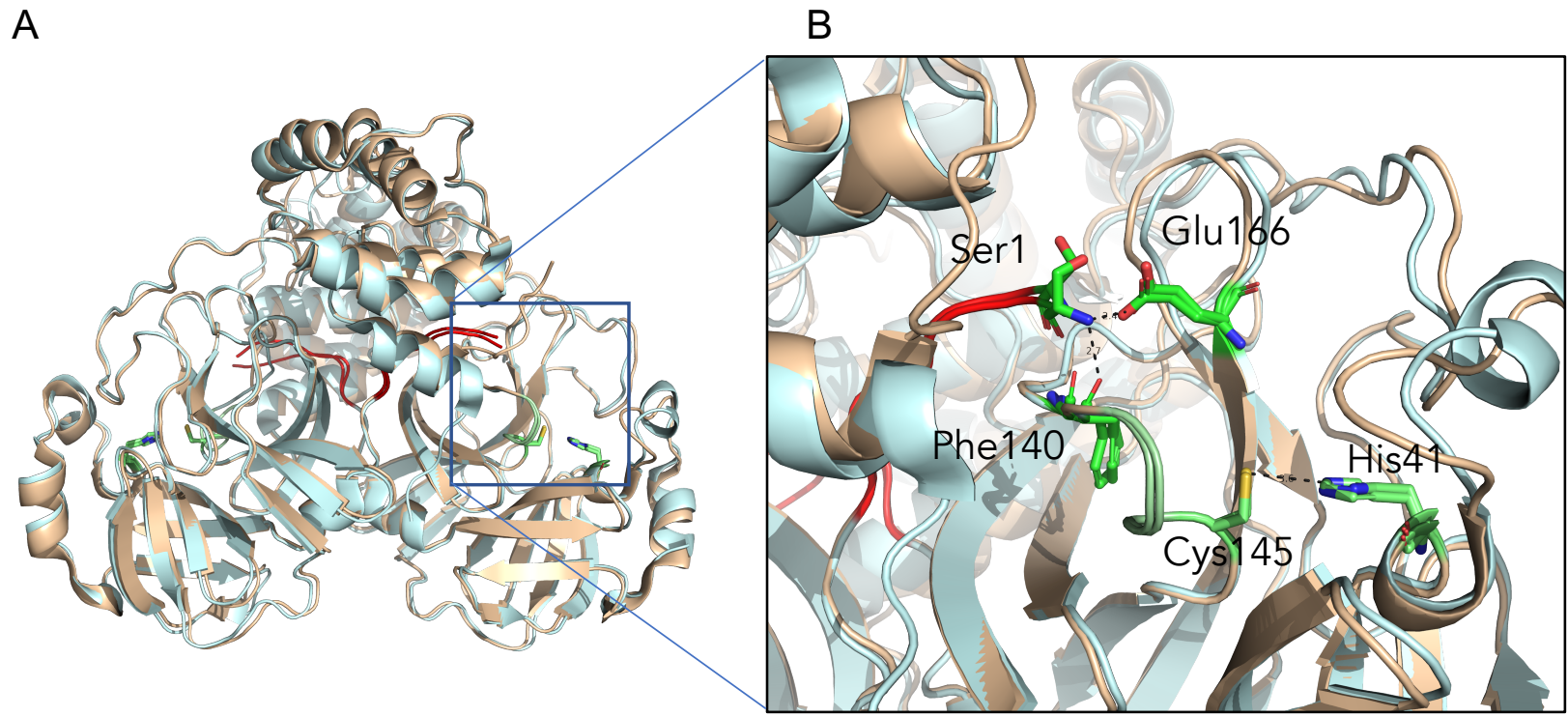

Figure 7. The N-terminus of one protomer interacts with the active site region of the other protomer. (A) Comparison of SARS-CoV M ${ }^{\text {pro }}$ (wheat) and SARS-CoV-2 M $^{\text {pro }}$ (cyan) structures reveals the N-terminus of each protomer (red) participates in domain swapping in the other protomer. (B) Hydrogen bonding with the N-terminal Ser1 occurs with the side chain of Glu166 and backbone oxygen of Phe140. This influences the region adjacent to the catalytic residues Cys145 and His41, and the oxyanion hole, colored in green. 
A
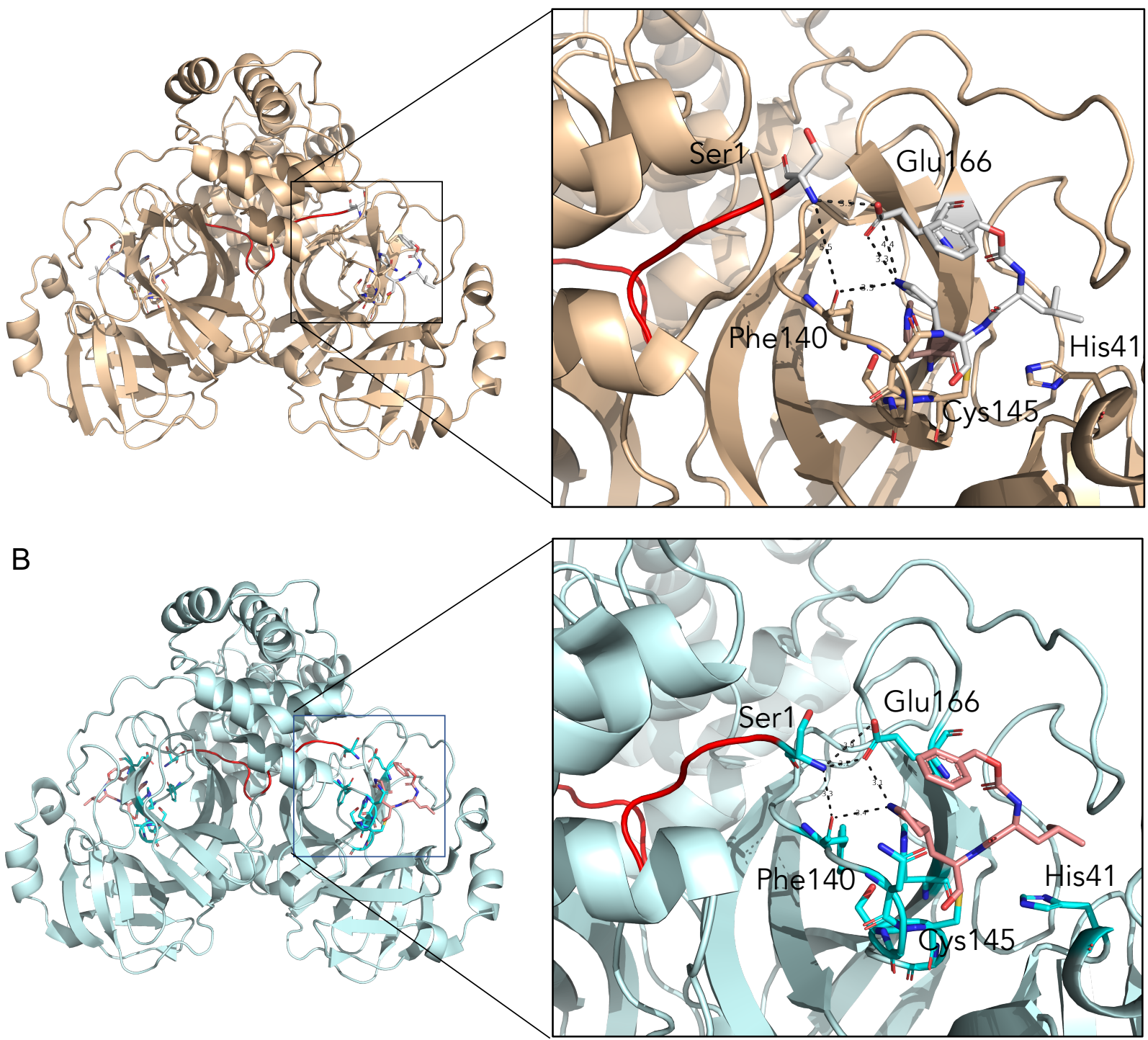

Figure 8 . The $\mathbf{N}$-terminal finger of the $M^{\text {pro }}$ stabilizes dimer formation and coordination of the drug GC373. The N-finger of $\mathrm{M}^{\text {pro }}$ facilitates coordination of drug GC373 in both in SARSCoV (7LCQ.PDB) (A) and SARS-CoV-2 (6WTJ.PDB) (B). Overall of 3-dimensional structures show the N-terminus (red) inserts into the second protomer in SARS-CoV $\mathrm{M}^{\text {pro }}$ (wheat) and in SARS-CoV-2 Mpro (cyan). Both residues that coordinate the N-finger, Phe140 and Glu166 also interact with the $\mathrm{P} 1$ position of the drug in $\mathrm{M}^{\text {pro }}$ of both SARS-CoV and SARS-CoV-2. 